\title{
GALOIS ACTIONS ON FUNDAMENTAL GROUPS OF CURVES AND THE CYCLE $C-C^{-}$
}

\author{
RICHARD HAIN AND MAKOTO MATSUMOTO
}

\section{INTRODUCTION}

The goal of this paper is to better understand the action of Galois groups on fundamental groups of smooth curves of genus 3 or more, and to show, using a fundamental result of Dennis Johnson [18, how the algebraic cycle $C-C^{-}$in the jacobian of a curve $C$ helps control the size of the image of the Galois group in the automorphism group of the curve's fundamental group.

Suppose that $K$ is a field of characteristic zero and that $X$ is a smooth projective variety defined over $K$. Denote $\operatorname{Gal}(\bar{K} / K)$ by $G_{K}$. Fix a prime number $\ell$. By standard constructions (Section 4.1), a codimension $r$ algebraic cycle $Z$ in $X$, defined over $K$ and homologically trivial in $X \otimes \bar{K}$, determines a class

$$
e_{Z} \in H^{1}\left(G_{K}, H_{\text {ét }}^{2 r-1}\left(X \otimes \bar{K}, \mathbb{Z}_{\ell}(r)\right)\right) \text {. }
$$

This depends only on the rational equivalence class of $Z$ and is the image of $Z$ under the $\ell$-adic Abel-Jacobi mapping

$$
C H_{\text {hom }}^{r}(X) \rightarrow H^{1}\left(G_{K}, H_{\text {ét }}^{2 r-1}\left(X \otimes \bar{K}, \mathbb{Z}_{\ell}(r)\right)\right)
$$

which is defined on the group of rational equivalence classes of homologically trivial, codimension $r$ cycles on $X$.

Suppose that $C$ is a smooth, geometrically connected projective curve over $K$ of genus $g \geq 3$ and that $\xi$ is a $K$-rational point of $C$. The morphism $\sigma_{\xi}: C \rightarrow \operatorname{Jac} C$ that takes $z \in C$ to the divisor class of $z-\xi$ is an embedding. Define the algebraic 1-cycle $C_{\xi}$ in Jac $C$ to be $\left(\sigma_{\xi}\right)_{*} C$. One also has the cycle $C_{\xi}^{-}:=i_{*} C_{\xi}$, where $i$ is the involution of the jacobian that takes each point to its inverse.

Two algebraic cycles particularly relevant to Galois actions on the fundamental group of the algebraic curve $(C, \xi)$ are:

(i) the 0 -cycle $(2 g-2) \xi-K_{C}$ in $C$, where $K_{C}$ is any canonical divisor of $C$;

(ii) the 1-cycle $C_{\xi}-C_{\xi}^{-}$in $\mathrm{Jac} C$.

Both are homologically trivial. The first cycle defines a class

$$
\kappa(C, \xi) \in H^{1}\left(G_{K}, H_{\text {ét }}^{1}\left(C \otimes \bar{K}, \mathbb{Z}_{\ell}(1)\right)\right)
$$

and the second a class

$$
\mu(C, \xi) \in H^{1}\left(G_{K}, H_{\text {ét }}^{2 g-3}\left(\operatorname{Jac} C \otimes \bar{K}, \mathbb{Z}_{\ell}(g-1)\right)\right) .
$$

Date: October 25, 2018.

1991 Mathematics Subject Classification. Primary 11G30; Secondary 14H30, 12G05, 14C25, $14 \mathrm{G} 32$.

Supported in part by grant DMS-0103667 from the National Science Foundation. 
It is convenient to set $H_{\mathbb{Z}_{\ell}}=H_{\text {ét }}^{1}\left(C \otimes \bar{K}, \mathbb{Z}_{\ell}(1)\right)$ and $L_{\mathbb{Z}_{\ell}}=\left(\Lambda^{3} H_{\mathbb{Z}_{\ell}}\right)(-1)$. Both are of weight -1 . Denote their tensor products with $\mathbb{Q}_{\ell}$ by $H_{\mathbb{Q}_{\ell}}$ and $L_{\mathbb{Q}_{\ell}}$, respectively. Wedging with the polarization $q \in \Lambda^{2} H_{\mathbb{Z}_{\ell}}(-1)$ defines a $G_{K}$-invariant embedding $H_{\mathbb{Z}_{\ell}} \rightarrow L_{\mathbb{Z}_{\ell}}$. Define

$$
\nu(C)=\text { the image of } \mu(C, \xi) \text { in } H^{1}\left(G_{K}, L_{\mathbb{Z}_{\ell}} / H_{\mathbb{Z}_{\ell}}\right) .
$$

This is independent of the choice of $\xi$ and is defined, even when $C$ has no $K$-rational points (cf. Section 10).

Suppose now that $K$ is a subfield of $\mathbb{C} .^{1}$ Let $\xi^{\text {an }}$ be the geometric point $\xi \otimes \mathbb{C}$ on the complex analytic space $C^{\text {an }}$ associated to $C$. Denote the Lie algebra of the $\mathbb{Q}_{\ell^{-}}$ form of the unipotent completion of the topological fundamental group $\pi_{1}\left(C^{\mathrm{an}}, \xi^{\mathrm{an}}\right)$ by $\mathfrak{p}(C, \xi)$. This is a pronilpotent Lie algebra over $\mathbb{Q}_{\ell}$.

Our goal in this paper is to describe how the classes $\nu(C), \mu(C, \xi)$ and $\kappa(C, \xi)$ influence the size of the image of $G_{K}$ in the automorphism group of $\mathfrak{p}(C, \xi)$. In particular, we are interested in the relationship between the image of $G_{K}$ in Aut $\mathfrak{p}(C, \xi)$ and the image of the mapping class group.

We shall denote the mapping class group of a closed, pointed, oriented surface $(S, x)$ by $\Gamma_{S, x}$. It is the group of connected components of the group of orientation preserving diffeomorphisms of $S$ that fix $x$ :

$$
\Gamma_{S, x}=\pi_{0} \operatorname{Diff}^{+}(S, x) .
$$

There is a natural homomorphism $\Gamma_{S, x} \rightarrow \operatorname{Aut} \pi_{1}(S, x)$. In the un-pointed case we shall denote the mapping class group of $S$ by $\Gamma_{S}$. It is the group of connected components of the group of orientation preserving diffeomorphisms of $S$ :

$$
\Gamma_{S}=\pi_{0} \operatorname{Diff}^{+} S \text {. }
$$

There is a natural homomorphism $\Gamma_{S} \rightarrow$ Out $\pi_{1}(S, x)$.

Denote the lower central series of $\mathfrak{p}(C, \xi)$ by

$$
\mathfrak{p}(C, \xi)=L^{1} \mathfrak{p}(C, \xi) \supset L^{2} \mathfrak{p}(C, \xi) \supset L^{3} \mathfrak{p}(C, \xi) \supset \cdots .
$$

The group Aut $\mathfrak{p}(C, \xi)$ is an affine proalgebraic group, as it is the inverse limit of the automorphism groups of the $\mathfrak{p}(C, \xi) / L^{m}$. The group $\pi_{1}\left(C^{\text {an }}, \xi^{\text {an }}\right)$ acts on itself by conjugation, and, by functoriality, on $\mathfrak{p}(C, \xi)$. Denote the Zariski closure of its image by $\operatorname{Inn} \mathfrak{p}(C, \xi)$. Set

$$
\text { Out } \mathfrak{p}(C, \xi)=\operatorname{Aut} \mathfrak{p}(C, \xi) / \operatorname{Inn} \mathfrak{p}(C, \xi) .
$$

This is also a proalgebraic group.

By functoriality, there are natural homomorphisms

$$
\theta_{C, \xi}: \Gamma_{C^{a n}, \xi^{\text {an }}} \rightarrow \operatorname{Aut} \mathfrak{p}(C, \xi) \text { and } \theta_{C}: \Gamma_{C^{\text {an }}} \rightarrow \operatorname{Out} \mathfrak{p}(C, \xi) .
$$

Since the filtration $L \bullet$ is by characteristic subalgebras, we also have the $m$-truncated representations

$$
\theta_{C, \xi}^{m}: \Gamma_{C^{\text {an }}, \xi^{\text {an }}} \rightarrow \text { Aut }\left[\mathfrak{p}(C, \xi) / L^{m+1}\right] \text { and } \theta_{C}^{m}: \Gamma_{C^{\text {an }}} \rightarrow \text { Out }\left[\mathfrak{p}(C, \xi) / L^{m+1}\right] .
$$

When $m=1$, these are the standard actions of $\Gamma_{C, \xi}$ and $\Gamma_{C}$ on the first homology of $C^{\text {an }}$.

\footnotetext{
${ }^{1}$ It is not really necessary to assume that $K$ is a subfield of $\mathbb{C}$, but we have chosen to do this in the hope of making the paper a little more accessible to non-experts. Experts should have no trouble generalizing our main results to arbitrary fields $K$ of characteristic zero and using standard arguments to deduce them from statements proved in this paper.
} 
On the other hand, the theory of algebraic fundamental groups gives nonabelian Galois representations

$$
\rho_{C, \xi}^{(\ell)}: G_{K} \rightarrow \operatorname{Aut} \pi_{1}^{(\ell)}\left(C^{\text {an }}, \xi^{\text {an }}\right) \text { and } \rho_{C}^{(\ell)}: G_{K} \rightarrow \text { Out } \pi_{1}^{(\ell)}\left(C^{\text {an }}, \xi^{\text {an }}\right),
$$

where $\pi_{1}^{(\ell)}\left(C^{\mathrm{an}}, \xi^{\mathrm{an}}\right)$ denotes the pro- $\ell$ completion of $\pi_{1}\left(C^{\mathrm{an}}, \xi^{\mathrm{an}}\right)$. These induce homomorphisms

$$
\rho_{C, \xi}: G_{K} \rightarrow \operatorname{Aut} \mathfrak{p}(C, \xi) \text { and } \rho_{C}: G_{K} \rightarrow \operatorname{Out} \mathfrak{p}(C, \xi) .
$$

We also have their $m$-truncated versions

$$
\rho_{C, \xi}^{m}: G_{K} \rightarrow \text { Aut }\left[\mathfrak{p}(C, \xi) / L^{m+1}\right] \text { and } \rho_{C}^{m}: G_{K} \rightarrow \text { Out }\left[\mathfrak{p}(C, \xi) / L^{m+1}\right]
$$

When $m=1$, these two homomorphisms both coincide with the Galois action on the abelianization of $\mathfrak{p}(C, \xi)$, which is canonically isomorphic to $H_{\mathbb{Q}_{\ell}}$. Since this action preserves the cup product up to a character, the image of $\rho_{C}^{1}$ is contained in the subgroup $\operatorname{GSp}\left(H_{\mathbb{Q}_{\ell}}\right)$ of Aut $H_{\mathbb{Q}_{\ell}}$. We will view $\rho_{C}^{1}$ as a homomorphism

$$
\rho_{C}^{1}: G_{K} \rightarrow \operatorname{GSp}\left(H_{\mathbb{Q}_{\ell}}\right) .
$$

The following theorem explains how the representation $\rho_{C}^{1}$ and the classes $\nu(C)$ and $\kappa(C, \xi)$ determine when the Zariski closure of the image of $G_{K}$ in Aut $\mathfrak{p}(C, \xi)$ contains the geometric automorphisms $\operatorname{im} \theta_{C, \xi}$. Recall that $C$ has genus $\geq 3$.

Theorem 1. If the $\ell$-adic cyclotomic character $G_{K} \rightarrow \mathbb{Z}_{\ell}^{\times}$has infinite image, then the following three conditions are equivalent:

(i) The Zariski closure of the image of $\rho_{C, \xi}$ contains the image of $\theta_{C, \xi}$.

(ii) The Zariski closure of the image of $\rho_{C, \xi}^{2}$ in Aut $\left[\mathfrak{p}(C, \xi) / L^{3}\right]$ contains the image of $\theta_{C, \xi}^{2}$.

(iii) The homomorphism $\rho_{C}^{1}: G_{K} \rightarrow \operatorname{GSp}\left(H_{\mathbb{Q}_{\ell}}\right)$ is Zariski dense, and both the classes $\kappa(C, \xi) \in H^{1}\left(G_{K}, H_{\mathbb{Z}_{\ell}}\right)$ and $\nu(C) \in H^{1}\left(G_{K}, L_{\mathbb{Z}_{\ell}} / H_{\mathbb{Z}_{\ell}}\right)$ have infinite order.

Remark 1.1. Several remarks are in order:

(a) This theorem has an alternative formulation, Theorem 9.1] in terms of pro- $\ell$ completions instead of unipotent completions.

(b) If one (and consequently all) of the conditions in the theorem are satisfied, then the Zariski closure of the image of $G_{K}$ in Aut $\mathfrak{p}(C, \xi)$ depends only on $g$, i.e., is independent of the choice of $(C, \xi)$.

(c) Matsumoto and Tamagawa 24, Thm. 1.2] prove a result which implies that there is an infinite number of genus $g$ curves defined over a number field that satisfy condition (i). Thus both classes $\kappa(C, \xi), \nu(C)$ are of infinite order for an infinite number of genus $g$ curves, a fact that can also be proved using the methods of Bloch-Esnault 2. Note that it is not known whether there is a number field $K$ for which there is an infinite number of curves with this property.

(d) One can use any homologically trivial cycle defined for each smooth curve $C$ whose normal function is a non-zero multiple of that of $C-C^{-}$. For example, one can use the Gross-Schoen cycle [7, which is a homologically trivial 1-cycle in the triple product of a curve of genus $\geq 3$ whose class in $H^{1}\left(G_{K}, L_{\mathbb{Z}_{\ell}}\right)$ is $3 \nu(C)$. 
(e) The theorem as stated does not hold in genus 2. However, there is a similar theorem for hyperelliptic curves (where $\xi$ is a Weierstrass point) which will be the subject of a future paper by the first author. The size of the Galois image in the hyperelliptic case is controlled by two cohomology classes which correspond to elements of Bloch's higher Chow groups. One part of the analogue of the Harris-Pulte Theorem needed in this case is the result of Colombo [5].

There is a similar result in the non-pointed case. In this case, the size of the Zariski closure of the image of $\rho_{C}$ is controlled by $\nu(C)$ and $\rho_{C}^{1}$. Note that the outer representations $\theta_{C}^{m}$ can be defined even when $C$ has no $K$-rational points (cf. Section [10]. So for this theorem, we need only assume that $C$ is a smooth projective curve of genus $\geq 3$ defined over the subfield $K$ of $\mathbb{C}$.

Theorem 2. If the $\ell$-adic cyclotomic character $G_{K} \rightarrow \mathbb{Z}_{\ell}^{\times}$has infinite image, then the following three conditions are equivalent:

(i) The Zariski closure of the image of $\rho_{C}$ contains the image of $\theta_{C}$.

(ii) The Zariski closure of the image of $\rho_{C}^{2}$ in Out $\left[\mathfrak{p}(C, \xi) / L^{3}\right]$ contains the image of $\theta_{C}^{2}$.

(iii) The homomorphism $\rho_{C}^{1}: G_{K} \rightarrow \operatorname{GSp}\left(H_{\mathbb{Q}_{\ell}}\right)$ is Zariski dense and the class $\nu(C)$ is of infinite order in $H^{1}\left(G_{K}, L_{\mathbb{Z}_{\ell}} / H_{\mathbb{Z}_{\ell}}\right)$.

Closely related to the density theorems above is the following $\ell$-adic analogue of the Harris-Pulte Theorem [29, p. 722], which we prove in Section 8

In Section 5.3 we show that the continuous action of a topological group $\Gamma$ on the pro- $\ell$ fundamental group $\pi_{1}^{(\ell)}\left(C^{\text {an }}, \xi^{\text {an }}\right)$ of $\left(C^{\text {an }}, \xi^{\text {an }}\right)$ determines a characteristic class in the continuous cohomology group $H^{1}\left(\Gamma, L_{\mathbb{Z}_{\ell}}\right)$. In particular, the action of $G_{K}$ on the pro- $\ell$ fundamental group of $\left(C^{\text {an }}, \xi^{\text {an }}\right)$ gives rise to a class

$$
m(C, \xi) \in H^{1}\left(G_{K}, L_{\mathbb{Z}_{\ell}}\right) .
$$

Theorem 3. If the genus of $C$ is $\geq 3$, then

$$
\mu(C, \xi)=m(C, \xi) \in H^{1}\left(G_{K}, L_{\mathbb{Z}_{\ell}}\right) .
$$

The original Harris-Pulte Theorem relates the mixed Hodge structure on $\pi_{1}(C, \xi)$ modulo the third term $L^{3}$ of its lower central series, where $C$ is a compact Riemann surface of genus $\geq 3$, to the element $\zeta(C, \xi)$ of

$$
\operatorname{Ext}_{\text {Hodge }}^{1}\left(\mathbb{Z}, \Lambda^{3} H^{1}\left(C^{\text {an }}, \mathbb{Z}\right)(1)\right)
$$

determined by $C_{\xi}-C_{\xi}^{-}$. The formulation [9] Thm. 11.1] asserts that $\zeta(C, \xi)=$ $2 \rho(C, \xi)$, where $\rho(C, \xi)$ is the extension class of the mixed Hodge structure on $\pi_{1}\left(C^{\mathrm{an}}, \xi^{\mathrm{an}}\right) / L^{3}$. Note that $2 \rho(C, \xi)$ corresponds to $m(C, \xi)$.

An un-pointed version of Theorem 3 is stated in Section 10.5

We shall prove these three theorems by specialization from the case of the universal curve over the moduli space $\mathcal{M}_{g}^{1}$ of pointed smooth projective curves of genus $g$. A basic tool is the weighted completion of topological groups, which we review in Section 7.3

\section{Notation, Conventions And Preliminaries}

Throughout $K$ will be a field of characteristic zero. In this article, all schemes, varieties and stacks will be defined (and of finite type) over $K$ unless otherwise 
noted. By a stack, we shall mean a Deligne-Mumford stack [6]. The category of schemes over a base scheme $B$ is a full subcategory of the category of stacks over $B$.

Often $K$ will be a subfield of $\mathbb{C}$. In this case, we shall denote the algebraic closure of $K$ in $\mathbb{C}$ by $\bar{K}$ and the analytic variety corresponding to a variety (resp. stack) $X$ over $K$ by $X^{\text {an }}$. It will be viewed as a topological space (resp. orbifold) in the complex topology. Each complex point $\xi$ of $X$ determines a point of $X^{\text {an }}$, which we shall denote by $\xi^{\text {an }}$.

The algebraic fundamental group of a stack (or scheme) $X$ with base point the geometric point $x$ will be denoted by $\pi_{1}^{\text {alg }}(X, x)$. We shall use the notation $\pi_{1}(M, x)$ to denote the fundamental group of the pointed topological space (or orbifold) $(M, x)$.

The profinite completion of a discrete group $\Gamma$ will be denoted by $\widehat{\Gamma}$ or $\Gamma^{\Upsilon}$. The pro- $\ell$ completion of a discrete or profinite group $\Gamma$ will be denoted by $\Gamma^{(\ell)}$. The pro- $\ell$ fundamental group functor will be denoted by $\pi_{1}^{(\ell)}$ in both the algebraic and topological cases.

Suppose that $F$ is a field of characteristic zero. The unipotent (or Malcev) completion over $F$ of the discrete group $\Gamma$ will be denoted by $\Gamma_{/ F}^{u n}$. If no field is mentioned, we will take $F$ to be $\mathbb{Q}$. The prounipotent fundamental group functor will be denoted by $\pi_{1}^{\text {un }}$.

Now suppose that $\ell$ is a rational prime and that $F$ is an $\ell$-adic field. The continuous unipotent completion over $F$ of the profinite group $\Gamma$ ([11, Appendix A.2]) will be denoted by $\Gamma_{/ F}^{u n}$. If no field is mentioned, we will take $F$ to be $\mathbb{Q}_{\ell}$. In 11, Thm. A.6] it is shown that if $\Gamma$ is a finitely generated discrete group, then there are natural isomorphisms

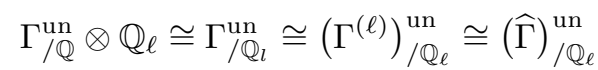

of $\mathbb{Q}_{\ell}$-prounipotent groups.

If $K$ is a subfield of $\mathbb{C}$, then for each variety $X$ over $K$ with an $\mathbb{C}$-rational point $x$, there is, by the standard comparison theorems in 8 and their generalizations, a canonical isomorphism

$$
\pi_{1}^{\text {alg }}(X \otimes \bar{K}, x) \cong \pi_{1}\left(X^{\text {an }}, x^{\text {an }}\right)^{\curlywedge} .
$$

This isomorphism also holds when $X$ is a stack and $X^{\text {an }}$ is regarded as an orbifold, 28, 32. It follows that there are natural isomorphisms

$$
\pi_{1}^{(\ell)}(X \otimes \bar{K}, x) \cong \pi_{1}^{(\ell)}\left(X^{\text {an }}, x^{\text {an }}\right) \text { and } \pi_{1}^{\text {un }}(X \otimes \bar{K}, x) \cong \pi_{1}^{\text {un }}\left(X^{\text {an }}, x^{\text {an }}\right) / \mathbb{Q}_{\ell} .
$$

When $G$ is a topological group and $V$ is a continuous $G$-module, $H^{\bullet}(G, V)$ will denote the continuous cohomology (in the sense of Tate 31]) of $G$ with coefficients in $V$.

\section{Monodromy Representations on Fundamental Groups}

3.1. Monodromy representations. Let $C \rightarrow B$ be a family of proper smooth pointed curves of genus $g$ defined over $K$. In our terminology, this means that $B$ is a stack, $C \rightarrow B$ is proper and smooth and endowed with a section $\xi: B \rightarrow C$, where each geometric fiber is a smooth, proper curve of genus $g$.

Assume that $B$ is geometrically connected. Let $x$ be a geometric point of $B$, and $C_{x}$ the fiber over $x$. Suppose that $g \geq 1$. Then we have an exact sequence of 
profinite groups

$$
1 \rightarrow \pi_{1}^{\mathrm{alg}}\left(C_{x}, \tilde{x}\right) \rightarrow \pi_{1}^{\mathrm{alg}}(C, \tilde{x}) \rightarrow \pi_{1}^{\mathrm{alg}}(B, x) \rightarrow 1,
$$

where $\tilde{x}=\xi(x)$.

The monodromy representation

$$
\rho_{C, x}^{\mathrm{alg}}: \pi_{1}^{\mathrm{alg}}(B, x) \rightarrow \operatorname{Aut} \pi_{1}^{\mathrm{alg}}\left(C_{x}, \tilde{x}\right)
$$

on the fundamental group associated to the data $C \rightarrow B, \xi$ and $x$ is defined to be the composite

$$
\pi_{1}^{\mathrm{alg}}(B, x) \stackrel{\xi_{*}}{\longrightarrow} \pi_{1}^{\mathrm{alg}}(C, \tilde{x}) \stackrel{\text { conjugation }}{\longrightarrow} \operatorname{Aut} \pi_{1}^{\mathrm{alg}}\left(C_{x}, \tilde{x}\right) .
$$

By functoriality, this homomorphism also induces continuous actions

$$
\rho_{C, x}^{(\ell)}: \pi_{1}^{\mathrm{alg}}(B, x) \rightarrow \operatorname{Aut} \pi_{1}^{(\ell)}\left(C_{x}, \tilde{x}\right), \quad \rho_{C, x}^{\mathrm{un}}: \pi_{1}^{\mathrm{alg}}(B, x) \rightarrow \operatorname{Aut} \pi_{1}^{\mathrm{un}}\left(C_{x}, \tilde{x}\right)
$$

of $\pi_{1}^{\text {alg }}(B, x)$ on the pro- $\ell$ and continuous unipotent completions, respectively, of $\pi_{1}^{\text {alg }}\left(C_{x}, \tilde{x}\right)$. In particular, when $B$ is Spec $K, \pi_{1}^{\text {alg }}(B, x)$ is $G_{K}$, the absolute Galois group of $K$, and these representations become the Galois representations

$$
\rho_{C, x}^{*}: G_{K} \rightarrow \operatorname{Aut} \pi_{1}^{*}\left(C_{x}, \tilde{x}\right)
$$

where $* \in\{\operatorname{alg},(\ell)$, un $\}$.

3.2. The universal monodromy representation. Suppose that $g \geq 1$. Denote the moduli stack of pointed smooth projective curves of genus $g>0$ over $K$ by $\mathcal{M}_{g}^{1}$. Denote the universal family over it by $\mathcal{C}_{g}^{1} \rightarrow \mathcal{M}_{g}^{1}$, and its tautological section by $\hat{\xi}: \mathcal{M}_{g}^{1} \rightarrow \mathcal{C}_{g}^{1}$. These are all defined over Spec $K$.

Each pointed curve $(C, x)$ defined over an algebraically closed extension of $K$ determines a geometric point $[C, x]$ of $\mathcal{M}_{g}^{1}$; the fiber of the pointed universal curve over this point may be identified with $(C, x)$. Associated to this geometric point is the monodromy representation

$$
\hat{\rho}_{C, x}^{\mathrm{alg}}: \pi_{1}^{\mathrm{alg}}\left(\mathcal{M}_{g}^{1},[C, x]\right) \rightarrow \operatorname{Aut} \pi_{1}^{\mathrm{alg}}(C, x) .
$$

This is called the universal monodromy representation for the pointed curve $(C, x)$ for reasons that we now recall.

For each proper family $C \stackrel{-\xi}{\longrightarrow} B$ of smooth pointed curves of genus $g$ defined over $K$, there is a unique morphism $[C, \xi]: B \rightarrow \mathcal{M}_{g}^{1}$, called the classifying morphism of the family, such that the pointed family

$$
C \stackrel{\xi}{\longrightarrow} B \quad \text { is the pullback of } \quad \mathcal{C}_{g}^{1} \stackrel{-\xi}{\longrightarrow} \mathcal{M}_{g}^{1}
$$

along $[C, \xi]$. It takes the geometric point $x$ of $B$ to $\left[C_{x}, \tilde{x}\right]$. By universality, the fiber of $\mathcal{C}_{g}^{1} \rightarrow \mathcal{M}_{g}^{1}$ over $[C, \xi](x)$ can be identified naturally with $\left(C_{x}, \tilde{x}\right)$. Thus, the diagram

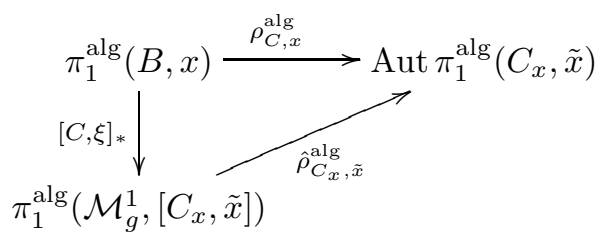


commutes, so that the monodromy representation of $C \rightarrow B$ associated to the geometric point $x$ of $B$ factors through the universal monodromy representation (4) associated to $\left(C_{x}, \tilde{x}\right)$.

By functoriality, the universal monodromy representation (4) also has pro- $\ell$ and prounipotent incarnations:

$$
\hat{\rho}_{C, x}^{(\ell)}: \pi_{1}^{\operatorname{alg}}\left(\mathcal{M}_{g}^{1},[C, x]\right) \rightarrow \operatorname{Aut} \pi_{1}^{(\ell)}(C, x)
$$

and

$$
\hat{\rho}_{C, x}^{\mathrm{un}}: \pi_{1}^{\mathrm{alg}}\left(\mathcal{M}_{g}^{1},[C, x]\right) \rightarrow \operatorname{Aut} \pi_{1}^{\mathrm{un}}(C, x)
$$

through which the representations (3) factor.

3.3. Geometric monodromy. Suppose that $(C, x)$ is a pointed curve defined over an algebraically closed extension field of $K$. This determines a geometric point $[C, x]$ of $\mathcal{M}_{g}^{1}$. It is standard that there is an exact sequence

$$
1 \rightarrow \pi_{1}^{\mathrm{alg}}\left(\mathcal{M}_{g}^{1} \otimes \bar{K},[C, x]\right) \rightarrow \pi_{1}^{\mathrm{alg}}\left(\mathcal{M}_{g}^{1},[C, x]\right) \rightarrow G_{K} \rightarrow 1 .
$$

The restriction

$$
\theta_{C, x}^{\mathrm{alg}}: \pi_{1}^{\mathrm{alg}}\left(\mathcal{M}_{g}^{1} \otimes \bar{K},[C, x]\right) \rightarrow \operatorname{Aut} \pi_{1}^{\mathrm{alg}}(C, x)
$$

of the universal monodromy (4) to the geometric fundamental group is called the geometric monodromy of the universal curve.

By functoriality, $\theta_{C, x}^{\text {alg }}$ induces representations

$$
\theta_{C, x}^{(\ell)}: \pi_{1}^{\mathrm{alg}}\left(\mathcal{M}_{g}^{1} \otimes \bar{K},[C, x]\right) \rightarrow \operatorname{Aut} \pi_{1}^{(\ell)}(C, x)
$$

and

$$
\theta_{C, x}^{\mathrm{un}}: \pi_{1}^{\mathrm{alg}}\left(\mathcal{M}_{g}^{1} \otimes \bar{K},[C, x]\right) \rightarrow \operatorname{Aut} \pi_{1}^{\mathrm{un}}(C, x) .
$$

Now suppose that $K$ is a subfield of $\mathbb{C}$ and that $(C, \xi)$ is a pointed curve defined over $K$. Let $\bar{K}$ be the algebraic closure of $K$ in $\mathbb{C}$. We regard $\mathcal{M}_{g}^{1 \text { an }}$ as an orbifold. There is a natural isomorphism

$$
\Gamma_{C^{\text {an }}, \xi^{\text {an }}} \cong \pi_{1}\left(\mathcal{M}_{g}^{1 \text { an }},\left[C^{\text {an }}, \xi^{\text {an }}\right]\right),
$$

which is unique up to conjugation by an element of $\operatorname{Aut}\left(C^{\text {an }}, \xi^{\text {an }}\right)$. Consequently, there is a natural isomorphism

$$
\widehat{\Gamma}_{C^{\text {an }}, \xi^{\text {an }}} \cong \pi_{1}^{\mathrm{alg}}\left(\mathcal{M}_{g}^{1} \otimes \bar{K},[\bar{C}, \bar{\xi}]\right),
$$

with the profinite completion of $\Gamma_{C^{\text {an }}, \xi^{\text {an }} \text {, where }} \bar{C}=C \otimes \bar{K}$ and $\bar{\xi}=\xi \otimes \bar{K}$. It is unique up to conjugation by an element of $\operatorname{Aut}\left(C^{\text {an }}, \xi^{\text {an }}\right)$.

By a result of Oda 28, with respect to these identifications, the geometric monodromy $\theta_{\bar{C}}, \bar{\xi}$ is the completion of the tautological action

$$
\Gamma_{C^{\text {an }}, \xi^{\text {an }}} \rightarrow \operatorname{Aut} \pi_{1}\left(C^{\text {an }}, \xi^{\text {an }}\right) .
$$

Remark 3.1. Suppose that $(C, \xi)$ is a pointed curve over $K$. Let $x$ be a geometric point of Spec K. A fundamental problem (cf. 24]) is to understand the image of 
the representation $\rho_{C, x}^{*}: G_{K} \rightarrow$ Aut $\pi_{1}^{*}\left(C_{x}, \tilde{x}\right)$, where $* \in\{\operatorname{alg},(\ell)$, un $\}$. Since the diagram

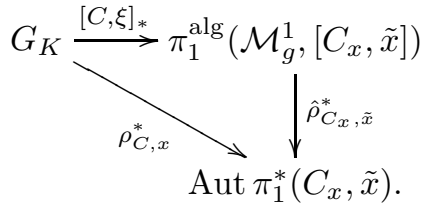

commutes, we have the inclusions

$$
\operatorname{im} \rho_{C, x}^{*} \hookrightarrow \operatorname{im} \hat{\rho}_{C_{x}, \tilde{x}}^{*} \hookrightarrow \text { Aut } \pi_{1}^{*}\left(C_{x}, \tilde{x}\right) .
$$

One can ask if the left hand inclusion can be an isomorphism. In 24, the second author and Tamagawa show that when $C_{x}$ is an affine curve of $g \neq 1$ and $*=\operatorname{alg}$, this inclusion is never an equality. More precisely, they prove that when $K$ is a number field, no nontrivial element of the geometric part $\theta_{C_{x}, \tilde{x}}^{\mathrm{alg}}$ lies in the image of $\rho_{C, x}^{\mathrm{alg}}$. On the other hand, when $*=(\ell)$, equality holds for the general curve (in the sense of Hilbert's Irreducibility Theorem). Theorem 1 treats the case $*=$ un, where image is replaced by the Zariski closure of the image.

\section{Cycle Classes}

In this section, we describe the class of the cycle class in the relative jacobian associated to a pointed family of curves $C \rightarrow B$.

4.1. The $\ell$-adic Abel-Jacobi map. Let $B$ be a geometrically connected stack over $K$, and let $f: X \rightarrow B$ be a proper smooth morphism locally of finite type. Let $\alpha$ be a relative algebraic cycle in $X / B$ of codimension $r$. That is, $\alpha$ is a formal integral linear combination of codimension- $r$ closed subvarieties of $X$ equidimensional over $B$. Denote the group of such relative cycles by $\operatorname{Cyc}^{r}(X / B)$.

One has the cycle class map

$$
c l: \operatorname{Cyc}^{r}(X / B) \rightarrow H_{\text {ét }}^{0}\left(B, R^{2 r} f_{*} \mathbb{Z}_{l}(r)\right) .
$$

When $K$ is a subfield of $\mathbb{C}$, we also have its analogue

$$
c l^{\text {an }}: \operatorname{Cyc}^{r}\left(X^{\text {an }} / B^{\text {an }}\right) \rightarrow H^{0}\left(B^{\text {an }}, R^{2 r} f_{*}^{\text {an }} \mathbb{Z}\right)
$$

for the associated analytic family $f^{\text {an }}: X^{\text {an }} \rightarrow B^{\text {an }} \operatorname{by~} \operatorname{Cyc}^{r}\left(X^{\text {an }} / B^{\text {an }}\right)$.

The kernels of these cycle maps are, by definition, the groups by $\mathrm{Cyc}_{\mathrm{hom}}^{r}(X / B)$ and $\mathrm{Cyc}_{\mathrm{hom}}^{r}\left(X^{\mathrm{an}} / B^{\mathrm{an}}\right)$, respectively, of homologically trivial cycles. Elements of the first group may be detected via the ( $\ell$-adic) Abel-Jacobi mapping

$$
A: \operatorname{Cyc}_{\mathrm{hom}}^{r}(X / B) \rightarrow H_{\text {ét }}^{1}\left(B, R^{2 r-1} f_{*} \mathbb{Z}_{l}(r)\right),
$$

and of the second by its topological analogue

$$
A^{\text {an }}: \operatorname{Cyc}_{\text {hom }}^{r}(X / B) \rightarrow H^{1}\left(B^{\text {an }}, R^{2 r-1} f_{*}^{\text {an }} \mathbb{Z}\right) .
$$

We now recall the definition of $A$.

Denote the support of an element $Z$ of $\operatorname{Cyc}_{\text {hom }}^{r}(X / B)$ by $|Z|$, and its inclusion into $X$ by $\iota:|Z| \hookrightarrow X$. Define $\mathcal{H}_{|Z|}^{2 r}\left(X, \mathbb{Z}_{\ell}(r)\right)$ to be the sheaf $\left(R^{2 r} \iota^{!}\right) \mathbb{Z}_{\ell}(r)$ over $B$, so that $\left(\left.f\right|_{|Z|}\right)_{*} \mathcal{H}_{|Z|}^{2 r}\left(X, \mathbb{Z}_{\ell}(r)\right)$ is the sheaf whose stalk at $b \in B$ is $H_{|Z|_{b}}^{2 r}\left(X_{b}, \mathbb{Z}_{\ell}(r)\right)$. Pulling back the Gysin sequence (cf. 25])

$$
0 \rightarrow R^{2 r-1} f_{*} \mathbb{Z}_{\ell}(r) \rightarrow R^{2 r-1}\left(\left.f\right|_{X-|Z|}\right)_{*} \mathbb{Z}_{\ell}(r)
$$




$$
\rightarrow\left(\left.f\right|_{|Z|}\right)_{*} \mathcal{H}_{|Z|}^{2 r}\left(X, \mathbb{Z}_{\ell}(r)\right) \rightarrow R^{2 r} f_{*} \mathbb{Z}_{\ell}(r)
$$

along the mapping $\left.\mathbb{Z}_{\ell} \rightarrow f\right|_{|Z|_{*}} \mathcal{H}_{|Z|}^{2 r}\left(X, \mathbb{Z}_{\ell}(r)\right)$ gives a short exact sequence

$$
0 \rightarrow R^{2 r-1} f_{*} \mathbb{Z}_{\ell}(r) \rightarrow \mathcal{E} \rightarrow \mathbb{Z}_{\ell} \rightarrow 0 .
$$

The class of this extension of smooth $\mathbb{Z}_{\ell}$-sheaves over $B$ in

$$
\operatorname{Ext}^{1}\left(\mathbb{Z}_{\ell}, R^{2 r-1} f_{*} \mathbb{Z}_{\ell}(r)\right) \cong H_{\text {ét }}^{1}\left(B, R^{2 r-1} f_{*} \mathbb{Z}_{\ell}(r)\right)
$$

is $A(Z)$.

In the case where $B$ is the spectrum of the field $K$, the Abel-Jacobi map is constant on rational equivalence classes of cycles, and therefore defines a map

$$
A: C H_{\text {hom }}^{r}(X / K) \rightarrow H_{\text {ét }}^{1}\left(K, R^{2 r-1} f_{*} \mathbb{Z}_{\ell}(r)\right) \cong H^{1}\left(G_{K}, H^{2 r-1}\left(X, \mathbb{Z}_{\ell}(r)\right)\right)
$$

which coincides with the usual $\ell$-adic Abel-Jacobi map (cf. [16, Lem. 9.4]).

When $K \subseteq \mathbb{C}$, the topological Abel-Jacobi mapping is defined in an analogous way. Standard comparison theorems imply that there is a commutative diagram

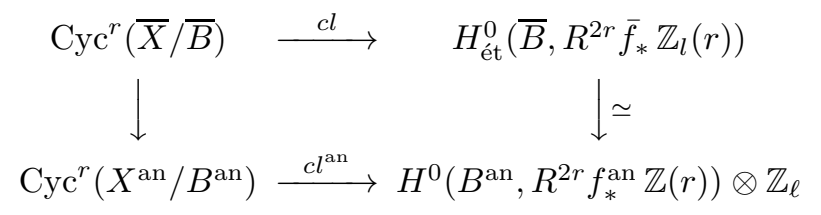

where $\bar{f}: \bar{X} \rightarrow \bar{B}$ is the pullback of $f: X \rightarrow B$ along $K \rightarrow \bar{K}$. Once again, standard comparison theorems imply the commutativity of the diagram

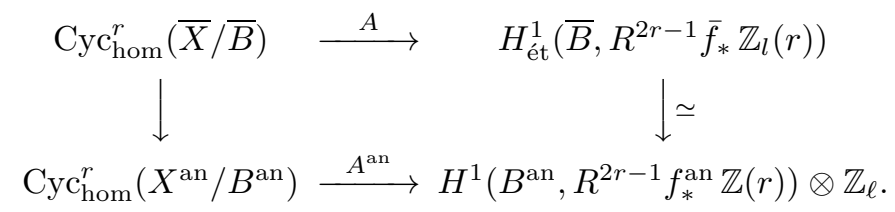

4.2. Setting and notation. Here we make precise the setting and notation we will use in much of the rest of the paper.

Suppose that $C \rightarrow B$ is a proper, geometrically connected, smooth family of curves of genus $g$ defined over $K$, where $g \geq 3$. Suppose that $\xi: B \rightarrow C$ is a section. Denote the relative jacobian $\operatorname{Jac}_{C / B}$ by $f: X \rightarrow B$. Suppose that $x$ is a geometric point of $B$.

For all such families, we shall denote the $\ell$-adic local system $R^{2 g-3} f_{*} \mathbb{Z}_{\ell}(g-1)$ over $B$ by $\mathbb{L}_{\mathbb{Z}_{\ell}}$. Similarly, denote the $\ell$-adic local system $R^{2 g-1} f_{*} \mathbb{Z}_{\ell}(g)$ over $B$ by $\mathbb{H}_{\mathbb{Z}_{\ell}}$. Note that $\mathbb{H}_{\mathbb{Z}_{\ell}}$ is the local system corresponding to the action of $\pi_{1}^{\text {alg }}(B, x)$ on the module $H_{\mathbb{Z}_{\ell}}:=H_{\text {ét }}^{1}\left(C_{x}, \mathbb{Z}_{\ell}(1)\right)$ and that $\mathbb{L}_{\mathbb{Z}_{\ell}}$ is the local system corresponding to the action of $\pi_{1}^{\text {alg }}(B, x)$ on the module $L_{\mathbb{Z}_{\ell}}:=\left[\Lambda^{3} H_{\mathbb{Z}_{\ell}}\right](-1)$.

If $B$ is geometrically connected and $x$ is a geometric point of $B$, then there are natural isomorphisms (cf. 25])

$$
H_{\text {ét }}^{1}\left(B, \mathbb{L}_{\mathbb{Z}_{\ell}}\right) \cong H^{1}\left(\pi_{1}^{\text {alg }}(B, x), L_{\mathbb{Z}_{\ell}}\right) \text { and } H_{\text {ét }}^{1}\left(B, \mathbb{H}_{\mathbb{Z}_{\ell}}\right) \cong H^{1}\left(\pi_{1}^{\text {alg }}(B, x), H_{\mathbb{Z}_{\ell}}\right) .
$$

When $K$ is a subfield of $\mathbb{C}$, we can associate to the corresponding analytic families $C^{\text {an }} \rightarrow B^{\text {an }}$ and $f^{\text {an }}: X^{\text {an }} \rightarrow B^{\text {an }}$ the local systems

$$
R^{2 g-3} f_{*}^{\text {an }} \mathbb{Z}(g-1) \text { and } R^{2 g-1} f_{*}^{\text {an }} \mathbb{Z}(g),
$$

which we shall denote by $\mathbb{L}_{\mathbb{Z}}$ and $\mathbb{H}_{\mathbb{Z}}$, respectively. 
4.3. The class $\mu(C, \xi)$. As in Section 1 we have two closed embeddings of $C$ in its Jacobian $X$; namely, $y \mapsto[y]-[\xi]$ and $y \mapsto[\xi]-[y]$. These define the two relative algebraic cycles

$$
C_{\xi}, C_{\xi}^{-} \in \mathrm{Cyc}^{g-1}(X / B),
$$

respectively. Denote their difference $C_{\xi}-C_{\xi}^{-}$by $\alpha(C, \xi)$. Since the endomorphism - id of $X$ multiplies $\alpha(C, \xi)$ by -1 and acts trivially on $R^{2} f_{*} \mathbb{Z}_{\ell}$, which is torsion free, it follows that $\alpha(C, \xi)$ is homologically trivial. It therefore determines a class

$$
A(\alpha(C, \xi)) \in H_{\text {ét }}^{1}\left(B, \mathbb{L}_{\mathbb{Z}_{\ell}}\right)
$$

which we shall denote by $\mu(C, \xi)$.

We will consider $\mu(C, \xi)$ as an element of $H^{1}\left(\pi_{1}^{\text {alg }}(B, x), L_{\mathbb{Z}_{\ell}}\right)$. This generalizes the definition given in 1

This class is functorial with respect to morphisms of pointed families. In particular, we have the class

$$
\mu\left(\mathcal{C}_{g}^{1}, \hat{\xi}\right) \in H_{\text {ét }}^{1}\left(\mathcal{M}_{g}^{1}, \mathbb{L}_{\mathbb{Z}_{\ell}}\right)
$$

of the universal curve, which is universal.

Proposition 4.1. If $C \stackrel{-\xi}{\longrightarrow} B$ is a pointed family over $K$ and $x$ is a geometric point of $B$, then

$$
\mu(C, \xi) \in H^{1}\left(\pi_{1}^{\text {alg }}(B, x), L_{\mathbb{Z}_{\ell}}\right)
$$

is the pullback of

$$
\mu\left(\mathcal{C}_{g}^{1}, \hat{\xi}\right) \in H^{1}\left(\pi_{1}^{\text {alg }}\left(\mathcal{M}_{g}^{1},\left[C_{x}, \tilde{x}\right]\right), L_{\mathbb{Z}_{\ell}}\right)
$$

under the group homomorphism induced by the classifying morphism

$$
[C, \xi]:(B, x) \rightarrow\left(\mathcal{M}_{g}^{1},\left[C_{x}, \tilde{x}\right]\right) .
$$

When $K$ is a subfield of $\mathbb{C}$, we have the relative cycle $C_{\xi^{\mathrm{an}}}^{\mathrm{an}}-C_{\xi^{\mathrm{an}}}^{\mathrm{an}-}$ in $f^{\mathrm{an}}: X^{\mathrm{an}} \rightarrow$ $B^{\text {an }}$. This determines a class

$$
\mu^{\text {an }}(C, \xi) \in H^{1}\left(B^{\text {an }}, \mathbb{L}_{\mathbb{Z}}\right) .
$$

Applying this construction to the universal pointed curve $\mathcal{C}_{g}^{\text {an } 1} \rightarrow \mathcal{M}_{g}^{\text {an } 1}$, we obtain the universal such class

$$
\mu^{\mathrm{an}}\left(\mathcal{C}_{g}^{1}, \hat{\xi}\right) \in H^{1}\left(\mathcal{M}_{g}^{\mathrm{an} 1}, \mathbb{L}_{\mathbb{Z}}\right) .
$$

Since the diagram (10) commutes and since this class is respected by base change, we have:

Proposition 4.2. The class $\mu^{\mathrm{an}}(C, \xi)$ corresponds to $\mu(C, \xi)$ under the comparison isomorphism

$$
H_{\text {ét }}^{1}\left(B \otimes \bar{K}, \mathbb{L}_{\mathbb{Z}_{\ell}}\right) \cong H_{\text {ét }}^{1}\left(B \otimes \mathbb{C}, \mathbb{L}_{\mathbb{Z}_{\ell}}\right) \cong H^{1}\left(B^{\text {an }}, \mathbb{L}_{\mathbb{Z}}\right) \otimes \mathbb{Z}_{\ell} .
$$

Moreover, the class $\mu^{\text {an }}(C, \xi)$ is the pullback of $\mu^{\text {an }}\left(\mathcal{C}_{g}^{\text {an } 1}, \hat{\xi}\right)$ along the classifying mapping $[C, \xi]: B^{\text {an }} \rightarrow \mathcal{M}_{g}^{\text {an } 1}$. 
4.4. The class $\kappa(C, \xi)$. Denote the canonical divisor class of $C$ over $B$ by $\mathcal{K}_{C}$. The cycle $(2 g-2) \xi-\mathcal{K}_{C}$ is homologous to zero on each geometric fiber of $C \rightarrow B$. It therefore defines a class

$$
\kappa(C, \xi) \in H_{\text {ét }}^{1}\left(B, \mathbb{H}_{\mathbb{Z}_{\ell}}\right) .
$$

Applying this construction to the universal pointed curve $\mathcal{C}_{g}^{1} \rightarrow \mathcal{M}_{g}^{1}$, we obtain the universal such class

$$
\kappa\left(\mathcal{C}_{g}^{1}, \hat{\xi}\right) \in H_{\text {ét }}^{1}\left(\mathcal{M}_{g}^{1}, \mathbb{H}_{\mathbb{Z}_{\ell}}\right) .
$$

As in the case of $\mu(C, \xi)$, this class pulls back to $\kappa(C, \xi)$ along the classifying morphism of the pointed family $C \rightarrow B$.

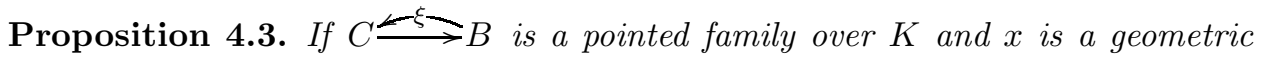
point of $B$, then

is the pullback of

$$
\kappa(C, \xi) \in H^{1}\left(\pi_{1}^{\text {alg }}(B, x), H_{\mathbb{Z}_{\ell}}\right)
$$

$$
\kappa\left(\mathcal{C}_{g}^{1}, \hat{\xi}\right) \in H^{1}\left(\pi_{1}^{\text {alg }}\left(\mathcal{M}_{g}^{1},\left[C_{x}, \tilde{x}\right]\right), H_{\mathbb{Z}_{\ell}}\right)
$$

under the group homomorphism induced by the classifying morphism

$$
[C, \xi]:(B, x) \rightarrow\left(\mathcal{M}_{g}^{1},\left[C_{x}, \tilde{x}\right]\right) .
$$

When $K$ is a subfield of $\mathbb{C}$, we have the relative cycle $(2 g-2) \xi^{\text {an }}-\mathcal{K}_{C}$ in $C \rightarrow B$. This determines a class

$$
\kappa^{\text {an }}(C, \xi) \in H^{1}\left(B^{\text {an }}, \mathbb{H}_{\mathbb{Z}}\right) .
$$

Applying this construction to the universal pointed curve $\mathcal{C}_{g}^{\text {an } 1} \rightarrow \mathcal{M}_{g}^{\text {an } 1}$, we obtain the universal such class

$$
\kappa^{\text {an }}\left(\mathcal{C}_{g}^{1}, \hat{\xi}\right) \in H^{1}\left(\mathcal{M}_{g}^{\text {an } 1}, \mathbb{H}_{\mathbb{Z}}\right) .
$$

The commutativity of the diagram (10) and naturality of the class under base change implies:

Proposition 4.4. The class $\kappa^{\text {an }}(C, \xi)$ corresponds to $\kappa(C, \xi)$ under the comparison isomorphism

$$
H_{\text {èt }}^{1}\left(B \otimes \bar{K}, \mathbb{H}_{\mathbb{Z}_{\ell}}\right) \cong H_{\text {ét }}^{1}\left(B \otimes \mathbb{C}, \mathbb{H}_{\mathbb{Z}_{\ell}}\right) \cong H^{1}\left(B^{\text {an }}, \mathbb{H}_{\mathbb{Z}}\right) \otimes \mathbb{Z}_{\ell} .
$$

Moreover, the class $\kappa^{\mathrm{an}}(C, \xi)$ is the pullback of $\kappa^{\mathrm{an}}\left(\mathcal{C}_{g}^{\mathrm{an} 1}, \hat{\xi}\right)$ along the classifying mapping $[C, \xi]: B^{\text {an }} \rightarrow \mathcal{M}_{g}^{\text {an } 1}$.

4.5. The classes $\nu(C)$ and $\tilde{\nu}(C, \xi)$. Denote by $\tilde{\nu}(C, \xi)$ the image of $\mu(C, \xi)$ under the quotient mapping

$$
H_{\text {ét }}^{1}\left(B, \mathbb{L}_{\mathbb{Z}}\right) \rightarrow H_{\text {ét }}^{1}\left(B, \mathbb{L}_{\mathbb{Z}} / \mathbb{H}_{\mathbb{Z}_{\ell}}\right) .
$$

Proposition 4.5. If $C \stackrel{-\xi}{\longrightarrow}$ is a pointed family over $K$ and $x$ is a geometric point of $B$, then

$$
\tilde{\nu}(C, \xi) \in H^{1}\left(\pi_{1}^{\mathrm{alg}}(B, x), L_{\mathbb{Z}_{\ell}} / H_{\mathbb{Z}_{\ell}}\right)
$$

is the pullback of

$$
\tilde{\nu}\left(\mathcal{C}_{g}^{1}, \hat{\xi}\right) \in H^{1}\left(\pi_{1}^{\text {alg }}\left(\mathcal{M}_{g}^{1},\left[C_{x}, \tilde{x}\right]\right), L_{\mathbb{Z}_{\ell}} / H_{\mathbb{Z}_{\ell}}\right)
$$

under the group homomorphism induced by the classifying morphism

$$
[C, \xi]:(B, x) \rightarrow\left(\mathcal{M}_{g}^{1},\left[C_{x}, \tilde{x}\right]\right) .
$$


When $K$ is a subfield of $\mathbb{C}$, define

$$
\tilde{\nu}^{\text {an }}(C, \xi) \in H^{1}\left(B^{\text {an }}, \mathbb{L}_{\mathbb{Z}_{\ell}} / \mathbb{H}_{\mathbb{Z}_{\ell}}\right),
$$

to be the image of $\mu^{\text {an }}(C, \xi)$ under the natural mapping

$$
H^{1}\left(B^{\mathrm{an}}, \mathbb{L}_{\mathbb{Z}}\right) \rightarrow H^{1}\left(B^{\mathrm{an}}, \mathbb{L}_{\mathbb{Z}_{\ell}} / \mathbb{H}_{\mathbb{Z}_{\ell}}\right) .
$$

Applying this construction to the universal pointed curve $\mathcal{C}_{g}^{\text {an } 1} \rightarrow \mathcal{M}_{g}^{\text {an } 1}$, we obtain the universal such class

$$
\tilde{\nu}^{\text {an }}\left(\mathcal{C}_{g}^{1}, \hat{\xi}\right) \in H^{1}\left(\mathcal{M}_{g}^{\text {an } 1}, \mathbb{L}_{\mathbb{Z}} / \mathbb{H}_{\mathbb{Z}}\right)
$$

Since the diagram (10) commutes and since the this class is respected by base change, we have:

Proposition 4.6. The class $\tilde{\nu}^{\text {an }}(C, \xi)$ corresponds to $\tilde{\nu}(C, \xi)$ under the comparison isomorphism

$$
H_{\text {ét }}^{1}\left(B \otimes \bar{K}, \mathbb{L}_{\mathbb{Z}_{\ell}} / \mathbb{H}_{\mathbb{Z}_{\ell}}\right) \cong H_{\text {ét }}^{1}\left(B \otimes \mathbb{C}, \mathbb{L}_{\mathbb{Z}_{\ell}} / \mathbb{H}_{\mathbb{Z}_{\ell}}\right) \cong H^{1}\left(B^{\text {an }}, \mathbb{L}_{\mathbb{Z}} / \mathbb{H}_{\mathbb{Z}}\right) \otimes \mathbb{Z}_{\ell}
$$

Moreover, the class $\tilde{\nu}^{\mathrm{an}}(C, \xi)$ is the pullback of $\tilde{\nu}^{\mathrm{an}}\left(\mathcal{C}_{g}^{\mathrm{an} 1}, \hat{\xi}\right)$ along the classifying mapping $[C, \xi]: B^{\text {an }} \rightarrow \mathcal{M}_{g}^{\text {an } 1}$.

Although we have used the rational point $\xi$ to define the class $\tilde{\nu}(C, \xi)$, this class depends only on $C$ and not on the rational point $\xi$ (or even the existence of the rational point). The following result is proved in Section 10.4

Proposition 4.7. The class $\tilde{\nu}(C, \xi)$ depends only on $C \rightarrow B$ and is independent of the choice or existence of the B-rational point $\xi$.

For this reason, we shall denote $\tilde{\nu}(C, \xi)$ by $\nu(C)$. Note, however, that our proof of Theorem 1 does not depend on this equality, and that Section 10.4 where this equality is proved, can be read at this point of the paper.

\section{The Universal Johnson Class}

In this section we construct a universal cohomology class

$$
\hat{m}^{(\ell)} \in H^{1}\left(\text { Aut } \pi_{1}^{(\ell)}(S, x), L_{\mathbb{Z}_{\ell}}\right)
$$

where $S$ is a compact Riemann surface of genus $g \geq 3$ and $L_{\mathbb{Z}_{\ell}}$ is the third exterior power of $H_{1}\left(S, \mathbb{Z}_{\ell}\right)$. This construction is a cohomological interpretation of an $\ell$-adic version of the Johnson homomorphism 17, which is, in turn, an outgrowth of a construction of Magnus.

The class $\hat{m}^{(\ell)}$ is a characteristic class for groups $G$ that act continuously on $\pi_{1}^{(\ell)}(S, x)$, such as Galois groups. Indeed, the class $\hat{m}^{(\ell)}$ can be pulled back along the homomorphism

$$
G \rightarrow \operatorname{Aut} \pi_{1}^{(\ell)}(S, x)
$$

that defines the action to give a class in $H^{1}\left(G, L_{\mathbb{Z}_{\ell}}\right)$. 
5.1. The Magnus homomorphism. Suppose that $\pi$ is a topological group. The examples we have in mind are where $\pi$ is either a discrete group (viewed as a topological group with the discrete topology) or a profinite group. Denote its group of continuous automorphisms by Aut $\pi$. We shall denote the lower central series filtration of $\pi$ by

$$
\pi=L^{1} \pi \supseteq L^{2} \pi \supseteq L^{3} \pi \supseteq \cdots
$$

where $L^{k+1} \pi$ is the closure of $\left[L, L^{k}\right]$.

Set $H_{1}(\pi)=\pi / L^{2} \pi$. Denote the kernel and image of the natural homomorphism

$$
\text { Aut } \pi \rightarrow \text { Aut } H_{1}(\pi)
$$

by $T$ and $R$, respectively, where $H_{1}(\pi)$ denotes the Hausdorff abelianization of $\pi$, which is the quotient of $\pi$ by the closure of its commutator subgroup. Since the filtration $L^{\bullet}$ of $\pi$ is by characteristic subgroups, there is a natural homomorphism

$$
\tilde{\epsilon}: T \rightarrow \operatorname{Hom}\left(H_{1}(\pi), \mathrm{Gr}_{L}^{2} \pi\right)
$$

into the group of continuous homomorphisms $H_{1}(\pi) \rightarrow \mathrm{Gr}_{L}^{2} \pi$. It is defined by taking the element $\phi$ of $T$ to the homomorphism

$$
u \mapsto \phi(\tilde{u}) u^{-1} \bmod L^{3} \pi
$$

where $\tilde{u} \in \pi$ is any lift of $u \in H_{1}(\pi)$. Since $\phi$ is continuous and acts trivially on $H_{1}(\pi)$, the homomorphism $\tilde{\epsilon}(u)$ is easily seen to be well defined and continuous. It is also easy to see that $\tilde{\epsilon}$ is itself a continuous homomorphism.

Each graded quotient of the lower central series of $\pi$ is naturally a continuous $R$-module. It is an exercise to show that the homomorphism

$$
\epsilon_{1}: H_{1}(T) \rightarrow \operatorname{Hom}\left(H_{1}(\pi), \mathrm{Gr}_{L}^{2} \pi\right)
$$

induced by $\tilde{\epsilon}$ is continuous and $R$-linear. We shall call it the Magnus homomorphism as Magnus 21] was the first to study the kernel of the homomorphism from the Automorphism group of a free group to the automorphisms of its abelianization. A similar construction was used by Andreadakis [1] when studying the automorphism group of a free. We shall view it as an element

$$
\epsilon_{1} \in \operatorname{Hom}_{R}\left(H_{1}(T), \operatorname{Hom}\left(H_{1}(\pi), \operatorname{Gr}_{L}^{2} \pi\right)\right),
$$

which we shall call the Magnus element.

It is well known that $\mathrm{Gr}_{L}^{\bullet} \pi$ is a graded Lie algebra (cf. 30). In our case, the bracket

$$
[, \quad]: \mathrm{Gr}_{L}^{n} \pi \otimes \mathrm{Gr}_{L}^{m} \pi \rightarrow \operatorname{Gr}_{L}^{n+m} \pi
$$

is easily seen to be $R$-equivariant.

The definition of $\epsilon_{1}$ can be extended to define continuous $R$-equivariant mappings

$$
\epsilon_{n}: H_{1}(T) \rightarrow \operatorname{Hom}\left(\mathrm{Gr}_{L}^{n} \pi, \mathrm{Gr}_{L}^{n+1} \pi\right)
$$

in such a way that, for each $\phi \in H_{1}(T),\left(\epsilon_{n}(\phi)\right)_{n} \in \mathrm{End}_{\mathrm{Gr}_{L}^{\bullet}} \pi$ is a derivation in the following sense.

Proposition 5.1. If $u \in \mathrm{Gr}_{L}^{i} \pi, v \in \mathrm{Gr}_{L}^{j} \pi$ and $i+j=n$, then for each $\phi \in H_{1}(T)$

$$
\epsilon_{n}(\phi)([u, v])=\left[\epsilon_{i}(\phi)(u), v\right]+\left[u, \epsilon_{j}(\phi)(v)\right] .
$$


5.2. The Johnson homomorphism. Now suppose that $\pi$ is either a hyperbolic surface group (that is, the fundamental group of a compact orientable surface of genus $g \geq 2$ ) with the discrete topology, or the pro- $\ell$ completion of such a surface group with the pro- $\ell$ topology. Let $A$ denote $\mathbb{Z}$ in the first case, and $\mathbb{Z}_{\ell}$ in the second. Then $H_{A}:=H_{1}(\pi)$ is a free $A$-module of rank $2 g$, which is endowed with a unimodular skew symmetric bilinear form $\omega$ - the intersection form. In this case, $R=\operatorname{GSp}\left(H_{A}\right)$, the group of symplectic similitudes of $H_{A}$. It is an extension

$$
1 \longrightarrow \mathrm{Sp}\left(H_{A}\right) \longrightarrow \mathrm{GSp}\left(H_{A}\right) \stackrel{\chi}{\longrightarrow} A^{\times} \longrightarrow 1
$$

of the units of $A$ by the symplectic group $\operatorname{Sp}\left(H_{A}\right)$. The character $\chi$ is the 1dimensional representation of GSp given by its action on the symplectic form $\omega$.

By a theorem of Labute [20], the Lie algebra $\mathrm{Gr}_{L}^{\bullet} \pi$ is torsion free as an $A$-module, and the natural surjection

$$
\operatorname{Lib}\left(H_{A}\right) \rightarrow \operatorname{Gr}_{L}^{\bullet} \pi
$$

induces a Lie algebra isomorphism

$$
\operatorname{Lib}\left(H_{A}\right) /(q) \cong \operatorname{Gr}_{L}^{\bullet} \pi
$$

induced by the isomorphism $H_{A} \rightarrow \operatorname{Gr}_{L}^{1} \pi$, where $\operatorname{Lib}(V)$ denotes the free Lie algebra over $A$ generated by the $A$-module $V$, and $q$ is the symplectic element of $\Lambda^{2} H_{A}$. In particular, the bracket $H_{A} \otimes H_{A} \rightarrow \mathrm{Gr}_{L}^{2} \pi$ is surjective and induces an $\operatorname{GSp}\left(H_{A}\right)$-equivariant isomorphism

$$
\operatorname{Gr}_{L}^{2} \pi \cong\left(\Lambda^{2} H_{1}(\pi)\right) / q .
$$

Denote the field of fractions of $A$ by $F$. That is $F=\mathbb{Q}$ when $A=\mathbb{Z}$ and $F=\mathbb{Q}_{\ell}$ when $A=\mathbb{Z}_{\ell}$. Then $H_{F}:=H_{A} \otimes F$ is the fundamental representation of $\operatorname{GSp}\left(H_{F}\right)$. Since the bracket

$$
H_{F} \otimes\left[\left(\mathrm{Gr}_{L}^{n} \pi\right) \otimes F\right] \rightarrow\left(\mathrm{Gr}_{L}^{n+1} \pi\right) \otimes F
$$

is surjective and $\operatorname{GSp}\left(H_{F}\right)$-equivariant for all $n \geq 1$, it follows by induction on $n$ that each

$$
\left(\operatorname{Gr}_{L}^{n} \pi\right) \otimes F
$$

is also a $\operatorname{GSp}\left(H_{F}\right)$-module.

Henceforth we shall regard $\operatorname{GSp}(H)$ as an algebraic group over $\mathbb{Z}$. Its group of $R$-rational points being $\operatorname{GSp}\left(H \otimes_{\mathbb{Z}} R\right)$. The action of this group on the symplectic form defines a homomorphism $\operatorname{GSp}(H) \rightarrow \mathbb{G}_{m}$. Denote the pullback of the standard representation of $\mathbb{G}_{m}$ by $\mathbb{Z}(1)$. Define $\mathbb{Z}(n)$ to be its $n$th tensor power when $n>0$, to be trivial when $n=0$, and to be the dual of $\mathbb{Z}(-n)$ when $n<0$. The tensor product of a representation $V$ of $\operatorname{GSp}(H)$ with $\mathbb{Z}(n)$ will be denoted by $V(n)$. (Later, this will correspond to Tate twist.) Since the pairing $H_{A} \otimes H_{A} \rightarrow A(1)$ is GSp-equivariant, so is its adjoint, the isomorphism

$$
H_{A}(-1) \cong \operatorname{Hom}\left(H_{A}, A\right) \text {. }
$$

In this case, the Magnus element induces a homomorphism

$$
\tilde{\tau}: H_{1}(T) \rightarrow H_{A}(-1) \otimes\left[\left(\Lambda^{2} H_{A}\right) / q\right] .
$$

Johnson made the further observation that when $\pi$ is a surface group, the Magnus homomorphism has restricted image. First note that there is a natural $\operatorname{GSp}\left(H_{A}\right)$ equivariant embedding

$$
f:\left[\Lambda^{3} H_{A}\right](-1) \rightarrow H_{A}(-1) \otimes\left[\left(\Lambda^{2} H_{A}\right) / q\right]
$$


defined by

$$
a \wedge b \wedge c \mapsto a \otimes(b \wedge c)+b \otimes(c \wedge a)+c \otimes(a \wedge b) .
$$

Lemma 5.2 (Johnson). The image of $\tilde{\tau}$ lies in the image of $f$.

Because of this, the Magnus homomorphism $\tilde{\tau}$ induces an $\operatorname{GSp}\left(H_{A}\right)$-equivariant homomorphism

$$
\tau: H_{1}(T) \rightarrow \Lambda^{3} H_{A}(-1)
$$

This is the celebrated Johnson homomorphism.

Since Johnson's Lemma is novel in the $\ell$-adic case, where $T$ is much larger than the Torelli group, and because we are applying it in such situations, we will give a proof. First we need:

Lemma 5.3. The sequence of $\operatorname{GSp}\left(H_{A}\right)$-modules

$$
0 \longrightarrow \Lambda^{3} H_{A} \stackrel{f(1)}{\longrightarrow} H_{A} \otimes \mathrm{Gr}_{L}^{2} \pi \stackrel{\text { bracket }}{\longrightarrow} \operatorname{Gr}_{L}^{3} \pi \longrightarrow 0
$$

is exact.

Proof. First, after tensoring the sequence with $F$, it is exact. This is well known (cf. [10, 8.3]) and easily proved using the representation theory of $\operatorname{Sp}\left(H_{F}\right)$. The right hand term is irreducible, and the left hand term is the sum of two irreducible components when $g \geq 3$ and is isomorphic to $H_{F}$ when $g=2$.

The result now follows over $A$ as, by Labute, each $\operatorname{Gr}_{L}^{n} \pi$ is torsion free, since the right hand mapping is surjective by the definition of the filtration $L^{\bullet}$, and since the kernel of the bracket divided by the image of $f$ is torsion free, which is easily checked by constructing an $A$-module splitting, for example.

Proof of Lemma 5.2. We use the fact that $H_{1}(T)$ acts on $\operatorname{Gr}_{L}^{\bullet} \pi$ as derivations. By Proposition [5.1] each $\phi \in T$ induces a map of short exact sequences

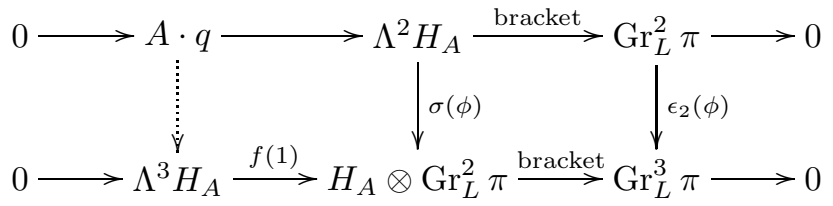

where $\sigma(\phi)(a \wedge b)=a \otimes \epsilon_{1}(\phi)(b)-b \otimes \epsilon_{1}(\phi)(a)$. Since the function $\epsilon_{1}(\phi): H_{A} \rightarrow$ $\mathrm{Gr}_{L}^{2} \pi$ corresponds, by duality, to

$$
\tilde{\tau}(\phi)=\sum_{j=1}^{g}\left(a_{j} \otimes \epsilon_{1}(\phi)\left(b_{j}\right)-b_{j} \otimes \epsilon_{1}(\phi)\left(a_{j}\right)\right) \in H_{A} \otimes \mathrm{Gr}_{L}^{2} \pi
$$

we see that $\tilde{\tau}(\phi)=\sigma(\phi)(q)$. The definitions then imply that

$$
\begin{aligned}
\text { bracket } \circ \sigma(\phi) & (q)=\text { bracket } \circ \sigma(\phi)\left(\sum_{j=1}^{g} a_{j} \wedge b_{j}\right) \\
& =\sum_{j=1}^{g}\left(\left[\epsilon_{1}(\phi)\left(a_{j}\right), b_{j}\right]-\left[a_{j}, \epsilon_{1}(\phi)\left(b_{j}\right)\right]\right)=\epsilon_{2}(\phi)\left(\sum_{j=1}^{g}\left[a_{j}, b_{j}\right]\right)=0 .
\end{aligned}
$$


5.3. A characteristic class for groups acting on surface groups. We retain the notation of the preceding paragraphs. In this paragraph, we explain how the Johnson homomorphism, or more accurately, twice the Johnson homomorphism, can be described as a cohomology class in

$$
H^{1}\left(\text { Aut } \pi, L_{A}\right),
$$

where $L_{A}=\left(\Lambda^{3} H_{A}\right)(-1)$. This can be viewed as a universal characteristic class of the group action.

One would like to say that the extension

$$
1 \rightarrow T \rightarrow \text { Aut } \pi \rightarrow \operatorname{GSp}\left(H_{A}\right) \rightarrow 1
$$

gives rise to a spectral sequence

$$
H^{s}\left(\operatorname{GSp}\left(H_{A}\right), H^{t}\left(T, L_{A}\right)\right) \Longrightarrow H^{s+t}\left(\text { Aut } \pi, L_{A}\right) \text {. }
$$

This is true in the discrete case, but may not be in the profinite case (cf. [15]). Nonetheless, one can easily establish, by a cocycle computation, the exactness of the sequence

$$
\begin{aligned}
& 0 \longrightarrow H^{1}\left(\operatorname{GSp}\left(H_{A}\right), L_{A}\right) \longrightarrow H^{1}\left(\text { Aut } \pi, L_{A}\right) \\
& \longrightarrow H^{0}\left(\operatorname{GSp}\left(H_{A}\right), H^{1}\left(T, L_{A}\right)\right) \stackrel{d_{2}}{\longrightarrow} H^{2}\left(\operatorname{GSp}\left(H_{A}\right), L_{A}\right)
\end{aligned}
$$

which one would have should the spectral sequence exist.

Note that the $\operatorname{GSp}\left(H_{A}\right)$-modules $L_{A}, H_{A}$ and $L_{A} / H_{A}$ all have the property that $-I \in \operatorname{GSp}\left(H_{A}\right)$ acts as - id on them.

Lemma 5.4. Suppose that $R_{A}$ is either $\operatorname{GSp}\left(H_{A}\right)$ or $\operatorname{Sp}\left(H_{A}\right)$ and that $g \geq 1$. If $V$ is an $R_{A}$-module on which $-I \in R_{A}$ acts as $-\mathrm{id}$, then the groups $H^{k}\left(R_{A}, V\right)$ have exponent 2 (that is, they are annihilated by 2). In particular, these groups vanish if $A=\mathbb{Z}_{\ell}$ when $\ell \neq 2$. If $V$ is torsion free, then there is a natural isomorphism

$$
H^{1}\left(R_{A}, V\right) \cong H^{0}\left(R_{A}, V / 2\right) \text {. }
$$

In particular, $H^{1}\left(R_{A}, L_{A}\right)$ vanishes for all $g \geq 1$.

Proof. The element $-I$ of the center of $R_{A}$ acts as - id on $H^{k}\left(R_{A}, L_{A}\right)$. But, by "center kills," elements of the center of $R_{A}$ act trivially on all cohomology groups of $R_{A}$. It follows that $H^{\bullet}\left(R_{A}, V\right)$ is annihilated by 2 . Since 2 is a unit of $\mathbb{Z}_{\ell}$ when $\ell \neq 2$, this implies the vanishing of $H^{\bullet}\left(R_{A}, L_{A}\right)$ in that case. It also implies the vanishing of $H^{0}\left(R_{A}, V\right)$ when $V$ is torsion free.

If $V$ is torsion free, the Bockstein sequence associated to

$$
0 \rightarrow V \stackrel{2}{\rightarrow} V \rightarrow V / 2 \rightarrow 0
$$

yields a natural isomorphism $\beta: H^{0}\left(R_{A}, V / 2\right) \rightarrow H^{1}\left(R_{A}, V\right)$.

When $g=1, L_{A}=0$, so that $H^{1}\left(R_{A}, L_{A}\right)$ vanishes. When $g=2$, cupping with the symplectic form gives a natural isomorphism $H_{A} \cong L_{A}$. One can easily verify that $H_{A} / 2$ has no invariants. Indeed, fix a curve $C$ of genus 2 . It is hyperelliptic. There are $2^{4}-1=15$ non-zero points of order 2 in the Jacobian of $C$. These are the 15 differences of 2 distinct Weierstrass points. Since none are fixed by the mapping class group, there are no invariants. The case $g \geq 3$ is treated in [14 $\S 6]$. There it is proved that, when $A=\mathbb{Z}, H^{0}\left(R_{A}, L_{A} / 2\right)$ vanishes. Since $L_{A}$ is torsion free and $H^{0}\left(R_{A}, L_{A} / 2\right)$ vanishes for all $g \geq 1, H^{1}\left(R_{A}, L_{A}\right)$ also vanishes. 
The following generalizes some results of Morita 27] from mapping class groups to the larger group Aut $\pi$.

Proposition 5.5. There is a unique class $\hat{m} \in H^{1}$ (Aut $\left.\pi, L_{A}\right)$ whose image in $H^{0}\left(\operatorname{GSp}\left(H_{A}\right), \operatorname{Hom}\left(H_{1}(T), L_{A}\right)\right)$ is $2 \tau$.

Proof. This follows from the facts that $H^{1}\left(\mathrm{GSp}\left(H_{A}\right), L_{A}\right)$ vanishes (Prop. 5.8) and that $H^{2}\left(\mathrm{GSp}\left(H_{A}\right), L_{A}\right)$ has exponent 2 (Lem. [5.4) using the exact sequence (12).

Remark 5.6. The image of $\tau$ under $d_{2}: H^{1}\left(\right.$ Aut $\left.\pi, L_{A}\right) \rightarrow H^{2}\left(\operatorname{GSp}\left(H_{A}\right), A\right)$ is nonzero when 2 is not a unit in $A$ (i.e., when $A=\mathbb{Z}_{2}$ and $\mathbb{Z}$ ). To prove this, it suffices to consider the case where $\pi$ is discrete and $A=\mathbb{Z}$ as there is a homomorphism Aut $\pi \rightarrow \operatorname{Aut} \pi^{(2)}$. In [27. p. 220], Morita proves the equivalent statement that $\tau$ is not in the image of

$$
H^{1}\left(\text { Aut } \pi, L_{\mathbb{Z}}\right) \rightarrow \operatorname{Hom}\left(H_{1}(T), L_{\mathbb{Z}}\right)
$$

but that $2 \tau$ is.

We shall adopt the following notation for the universal class in the $\ell$-adic case:

$$
\hat{m}^{(\ell)} \in H^{1}\left(\operatorname{Aut} \pi^{(\ell)}, L_{\mathbb{Z}_{\ell}}\right) .
$$

Definition 5.7. Suppose that $(C, \xi)$ is a pointed curve over $K$ of genus $g \geq 2$, where $K$ is a subfield of $\mathbb{C}$. Define the class

$$
m(C, x) \in H^{1}\left(G_{K}, L_{\mathbb{Z}_{\ell}}\right)
$$

to be the pullback of the universal class $\hat{m}^{(\ell)}$ along the homomorphism

$$
\rho_{C, x}^{(\ell)}: G_{K} \rightarrow \operatorname{Aut} \pi_{1}^{(\ell)}\left(C^{\text {an }}, \xi^{\text {an }}\right) .
$$

This is the invariant of the Galois action on the fundamental group that appears in the statement of the $\ell$-adic Harris-Pulte Theorem in the Introduction.

5.4. Further torsion computations. In this section Lemma 5.4 is extended to obtain more torsion vanishing examples that are needed in subsequent sections.

Wedging with the symplectic element $q$ of $H_{A}$ defines a $\operatorname{GSp}\left(H_{A}\right)$-invariant imbedding

$$
i: H_{A} \hookrightarrow L_{A} .
$$

There is also a $\operatorname{GSp}\left(H_{A}\right)$-invariant projection $c: L_{A} \rightarrow H_{A}$ defined by

$$
c: x \wedge y \wedge z \mapsto \omega(x, y) z+\omega(y, z) x+\omega(z, x) y
$$

It is easy to verify that $c \circ i=(g-1)$ id so that, when $g-1$ is a unit in $A$, we have a $\operatorname{GSp}\left(H_{A}\right)$-module decomposition

$$
(c, p): L_{A} \stackrel{\simeq}{\longrightarrow} H_{A} \oplus L_{A} / H_{A},
$$

where $p$ denotes the projection $L_{A} \rightarrow L_{A} / H_{A}$.

When $A$ is a field of characteristic zero, $H_{A}$ and $L_{A} / H_{A}$ are irreducible representations of the algebraic group $\operatorname{Sp}\left(H_{A}\right)$.

Proposition 5.8. If $R_{A}$ is $\operatorname{GSp}\left(H_{A}\right)$ or $\operatorname{Sp}\left(H_{A}\right)$, then the groups $H^{1}\left(R_{A}, H_{A}\right)$, $H^{1}\left(R_{A}, L_{A}\right)$ and $H^{1}\left(R_{A}, L_{A} / H_{A}\right)$ all vanish for all $g \geq 1$. 
Proof. In view of Lemma 5.4 we need only show that $H^{0}\left(R_{A}, V / 2\right)$ vanishes when $V=H_{A}$ and $L_{A} / H_{A}$. It is easy to check (and well known) that $H^{0}\left(R_{A}, H_{A} / 2\right)$ vanishes for all $g \geq 1$. Indeed, when $g=1$ and $A=\mathbb{Z}$ or $\mathbb{Z}_{2}$, then the image of $R_{A}$ in $\operatorname{Aut}\left(H_{A} / 2\right)$ is $S L_{2}\left(\mathbb{F}_{2}\right)$ and $H_{A} / 2$ is the two dimensional vector space over $\mathbb{F}_{2}$. The result follows in this case. When $g>1$, one can restrict to the subgroup $R^{\prime}$ of $R_{A}$ that is a product of $g$ copies of $S L_{2}(A)$. Then the image of $R^{\prime}$ in $\operatorname{Aut}\left(H_{A} / 2\right)$ is the product of $g$ copies of $S L_{2}\left(\mathbb{F}_{2}\right)$, and as an $R^{\prime}$-module, $H_{A}$ is the direct sum of $g$-copies of the 2-dimensional vector space over $\mathbb{F}_{2}$, from which it follows that $H^{0}\left(R_{A}, H_{A} / 2\right)$ vanishes, as claimed. Thus $H^{1}\left(R_{A}, H_{A}\right)$ vanishes.

The case $V=L_{A} / H_{A}$ follows from the previous case as $L_{A} / H_{A}$ can be imbedded in $L_{A}$ as an $R_{A}$-module. The mapping is defined by

$$
x \wedge y \wedge z+H_{A} \mapsto(g-1) x \wedge y \wedge z-q \wedge c(x \wedge y \wedge z)
$$

where $q$ denotes the symplectic element and $c$ is the contraction defined above. It is not difficult to show that this mapping is well defined and that its composition

$$
L_{A} / H_{A} \rightarrow L_{A} \rightarrow L_{A} / H_{A}
$$

with the quotient mapping is $g-1$ times the identity. Since $L_{A} / H_{A}$ is torsion free and the sequence

$$
0 \rightarrow L_{A} / H_{A} \rightarrow L_{A} \stackrel{c}{\rightarrow} H_{A} \rightarrow 0
$$

is exact, it follows (by tensoring with $\left.\mathbb{F}_{2}\right)$ that $\left(L_{A} / H_{A}\right) / 2$ injects into $L_{A} / 2$ from which it follows that $H^{0}\left(R_{A}, L_{A} / H_{A}\right)$ vanishes, as required.

\section{Topological Computations}

6.1. Johnson's Theorem. Suppose that $(S, x)$ is a pointed, compact oriented surface of genus $g \geq 2$. As in previous sections, we denote $H_{1}(S, A)$ by $H_{A}$ where $A$ is a ring such as $\mathbb{Z}, \mathbb{Z}_{\ell}, \mathbb{Q}, \mathbb{Q}_{\ell}$, and $\left(\Lambda^{3} H_{A}\right)(-1)$ by $L_{A}$.

The Torelli group $T_{S, x}$ is, by definition, the kernel of the natural homomorphism $\Gamma_{S, x} \rightarrow \mathrm{Sp}\left(H_{A}\right)$. By the preceding section, one has a Johnson homomorphism

$$
\tau_{S, x}: H_{1}\left(T_{S, x}\right) \rightarrow L_{\mathbb{Z}}
$$

Theorem 6.1 (18). If $g \geq 3$, the Johnson homomorphism $\tau_{S, x}$ is surjective and has finite kernel of exponent 2.

6.2. Consequences. Suppose that $V$ is an $\operatorname{Sp}\left(H_{\mathbb{Z}}\right)$-module. The spectral sequence

$$
H^{s}\left(\operatorname{Sp}\left(H_{\mathbb{Z}}\right), H^{t}\left(T_{S, x}\right) \otimes V\right) \Longrightarrow H^{s+t}\left(\Gamma_{S, x}, V\right)
$$

associated to the group extension

$$
1 \rightarrow T_{S, x} \rightarrow \Gamma_{S, x} \rightarrow \mathrm{Sp}\left(H_{\mathbb{Z}}\right) \rightarrow 1
$$

yields the exact sequence

$$
\begin{aligned}
0 \rightarrow H^{1}\left(\mathrm{Sp}\left(H_{\mathbb{Z}}\right), V\right) & \rightarrow H^{1}\left(\Gamma_{S, x}, V\right) \\
& \rightarrow H^{0}\left(\mathrm{Sp}\left(H_{\mathbb{Z}}\right), \operatorname{Hom}_{\mathbb{Z}}\left(H_{1}\left(T_{S, x}\right), V\right)\right) \rightarrow H^{2}\left(\operatorname{Sp}\left(H_{\mathbb{Z}}\right), V\right) .
\end{aligned}
$$

The modules $H_{\mathbb{Q}}$ and $L_{\mathbb{Q}}$ are rational representations of the algebraic group $\operatorname{Sp}\left(H_{\mathbb{Q}}\right)$. The representations $H_{\mathbb{Q}}$ and $L_{\mathbb{Q}} / H_{\mathbb{Q}}$ are irreducible and are the first and third fundamental representations, respectively. 
Proposition 6.2 (9. Prop. 5.2]). If $g \geq 3$ and $V$ is a finite dimensional irreducible representation of $\operatorname{Sp}\left(H_{\mathbb{Q}}\right)$, then $H^{1}\left(\Gamma_{S, x}, V\right)$ vanishes unless $V$ is isomorphic to either $H_{\mathbb{Q}}$ or $L_{\mathbb{Q}} / H_{\mathbb{Q}}$. In these cases we have

$$
H^{1}\left(\Gamma_{S, x}, H_{\mathbb{Q}}\right) \cong \mathbb{Q} \text { and } H^{1}\left(\Gamma_{S, x}, L_{\mathbb{Q}} / H_{\mathbb{Q}}\right) \cong \mathbb{Q} .
$$

Proof. This follows immediately from Johnson's Theorem (Thm. 6.1), the decomposition (14), the sequence (15), Schur's Lemma, and the fact that $H^{j}\left(\operatorname{Sp}\left(H_{\mathbb{Q}}\right), V\right)$ vanishes for $j=1,2$ by a Theorem of Borel [4] (cf. [10, Thm. 3.2]).

The following computations are equivalent to those of Morita in [27].

Corollary 6.3. If $g \geq 3$, we have $H^{1}\left(\Gamma_{S, x}, H_{\mathbb{Z}}\right) \cong \mathbb{Z}, \quad H^{1}\left(\Gamma_{S, x}, L_{\mathbb{Z}} / H_{\mathbb{Z}}\right) \cong \mathbb{Z}$ and $H^{1}\left(\Gamma_{S, x}, L_{\mathbb{Z}}\right) \cong \mathbb{Z}^{2}$.

Proof. It follows from Proposition [5.8 using the exact sequence (15), that the mapping

$$
H^{1}\left(\Gamma_{S, x}, V\right) \rightarrow H^{0}\left(\operatorname{Sp}\left(H_{\mathbb{Z}}\right), \operatorname{Hom}_{\mathbb{Z}}\left(H_{1}\left(T_{S, x}\right), V\right)\right)
$$

is injective when $V=H_{\mathbb{Z}}, L_{\mathbb{Z}}$ and $L_{\mathbb{Z}} / H_{\mathbb{Z}}$. Since the right hand group is torsion free, it follows that for these $V, H^{1}\left(\Gamma_{S, x}, V\right)$ is a torsion free $\mathbb{Z}$-module of rank equal to the dimension of $H^{1}\left(\Gamma_{S, x}, V \otimes \mathbb{Q}\right)$. The result now follows from the previous result.

Denote by $\hat{m}^{\text {an }}$ the unique element of $H^{1}\left(\Gamma_{S, x}, L_{\mathbb{Z}}\right)$ whose image in

$$
\operatorname{Hom}_{\mathrm{Sp}\left(H_{\mathbb{Z}}\right)}\left(H_{1}\left(T_{S, x}\right), L_{\mathbb{Z}}\right)
$$

is twice the Johnson homomorphism $2 \tau_{S, x}$. (Cf. Proposition 5.5)

6.3. A monodromy computation. In this paragraph, we give a detailed proof of the following assertion which is crucial in this paper, and whose proof is only sketched in 9].

Proposition 6.4. The classes $\hat{m}^{\text {an }}$ and $\mu^{\text {an }}\left(\mathcal{C}_{g}^{1}, \hat{\xi}\right)$ of $H^{1}\left(\mathcal{M}_{g}^{1 \text { an }}, \mathbb{L}_{\mathbb{Z}}\right)$ are equal.

Proof. Denote $\mu^{\text {an }}\left(\mathcal{C}_{g}^{1}, \hat{\xi}\right)$ by $\mu^{\text {an }}$. It follows from the injectivity of $H^{1}\left(\Gamma_{S, x}, L_{\mathbb{Z}}\right) \rightarrow$ $H^{0}\left(S, \operatorname{Hom}_{\mathbb{Z}}\left(H_{1}\left(T_{S, x}\right), L_{\mathbb{Z}}\right)\right)$ that to prove the result, we need only check the equality of $\hat{m}^{\text {an }}$ and $\mu^{\text {an }}$ in $\operatorname{Hom}\left(H_{1}\left(T_{C^{\text {an }}}, \xi^{\text {an }}\right), L_{\mathbb{Z}}\right)$. We do this by showing that the image of $\mu^{\text {an }}$ is $2 \tau_{S, x}$.

Fix a pointed compact Riemann surface $(S, x)$ as a base point of $\mathcal{M}_{g}^{1 \text { an }}$. Each $\phi \in T_{S, x}$ can be represented by an orbifold imbedding

$$
\Phi:\left(S^{1}, 1\right) \rightarrow\left(\mathcal{M}_{g}^{1 \text { an }},[C, x]\right) .
$$

The pullback of the universal curve along $\Phi$ is the mapping torus

$$
M(\phi)=(S \times[0,1]) /\{(\phi(s), 0) \sim(s, 1): s \in S\}
$$

which projects to $S^{1}$, which we identify with the unit interval with its ends identified. The universal section $\hat{\xi}$ pulls back to the section that takes $t \in[0,1] /\{0 \sim 1\}$ to $(x, t) \in M(\phi)$.

As a real manifold, the jacobian of $S$ is $H_{1}(S, \mathbb{R} / \mathbb{Z})$, which we shall denote by $J$. Since $\phi$ acts trivially on $H_{1}(S)$, the bundle of jacobians has a natural trivialization over $S^{1}$. The imbedding of the universal curve into the universal jacobian given 
by $\hat{\xi}$ restricts to an imbedding ${ }^{2} M(\phi) \rightarrow S^{1} \times J$ over $S^{1}$. Identify $M(\phi)$ with its image. The cycle $\mathcal{C}_{g, \hat{\xi}}-\mathcal{C}_{g, \hat{\xi}}^{-}$restricts to the topological 3-cycle

$$
Z:=M(\phi)-M(\phi)^{-}
$$

in $S^{1} \times J$ over $S^{1}$. Denote the fiber of $Z$ over $t \in S^{1}$ by $Z_{t}$.

By duality, the local system used to define the invariant $\mu^{\text {an }}\left(\mathcal{C}_{g}^{1}, \hat{\xi}\right)$ pulls back to the local system over $S^{1}$ whose fiber over $t$ is $H_{3}\left(J, Z_{t}\right)$. This is an extension

$$
0 \rightarrow H_{3}(J) \rightarrow H_{3}\left(J, Z_{t}\right) \rightarrow \mathbb{Z} \rightarrow 0
$$

in which a relative cycle whose boundary is $Z_{t}$ projects to 1 . The monodromy of this local system is trivial on $L_{\mathbb{Z}}$ and $\mathbb{Z}$, and is therefore given by a homomorphism $\mathbb{Z} \rightarrow H_{3}(J)$, which is, in turn, given by an element of $H_{3}(J)$. We will show that this invariant is

$$
2 p_{*}[M(\phi)] \in H_{3}(J) \cong L_{\mathbb{Z}}
$$

where $p: S^{1} \times J \rightarrow J$ is the projection. The result will then follow from Johnson's assertion [19] that

which is proved in 9 .

$$
p_{*}[M(\phi)]=\tau_{S, x}(\phi),
$$

We first work in the pullback $[0,1] \times J \rightarrow[0,1]$ along $[0,1] \rightarrow S^{1}$. Choose a 3 -chain $W_{0}$ in $J$ such that $\partial W_{0}=Z_{0}$. Let $Z_{[0, t]}$ be the restriction of $Z$ to $[0, t]$. Note that

$$
\partial Z_{[0, t]}=Z_{t}-Z_{0} .
$$

If we set $\widetilde{W}_{t}=W_{0}+Z_{[0, t]}$, then $\partial \widetilde{W}_{t}=Z_{t}$. To get a 3-chain in $J$, set $W_{t}=p_{*} \widetilde{W}_{t}$, where $p:[0,1] \times J \rightarrow J$ is the projection. Then $W_{t}$ is a 3 -chain in $J$ such that $\partial W_{t}=Z_{t}$. The monodromy is now the class of

$$
W_{1}-W_{0}=p_{*}\left(\widetilde{W}_{1}-\widetilde{W}_{0}\right)=p_{*}\left(Z_{[0,1]}\right)=p_{*}(M(\phi))-p_{*}\left(M(\phi)^{-}\right)
$$

in $H_{3}(J)$, which is $2 p_{*}([M(\phi)])$, as claimed.

6.4. Generators. In this section we show that several of the universal classes defined in Section 4 turn out to be integral generators of the cohomology groups in which they lie. The following result is closely related to computations of Morita in [27] and [26] where he identifies generators of $H^{1}\left(\Gamma_{S, x}, V\right)$ where $V$ is $H_{\mathbb{Z}}$ and $L_{\mathbb{Z}} / H_{\mathbb{Z}}$.

Proposition 6.5. If $g \geq 3$, then

(i) $\tilde{\nu}^{\text {an }}\left(\mathcal{C}_{g}^{1}, \hat{\xi}\right)$ freely generates $H^{1}\left(\mathcal{M}_{g}^{1 \text { an }}, \mathbb{L}_{\mathbb{Z}} / \mathbb{H}_{\mathbb{Z}}\right)$;

(ii) $\kappa^{\text {an }}\left(\mathcal{C}_{g}^{1}, \hat{\xi}\right)$ freely generates $H^{1}\left(\mathcal{M}_{g}^{1}\right.$ an, $\left.\mathbb{H}_{\mathbb{Z}}\right)$;

(iii) $\tilde{\nu}\left(\mathcal{C}_{g}^{1}, \hat{\xi}\right)$ freely generates $H_{\text {ét }}^{1}\left(\mathcal{M}_{g}^{1} \otimes \bar{K}, \mathbb{L}_{\mathbb{Z}_{\ell}} / \mathbb{H}_{\mathbb{Z}_{\ell}}\right)$;

(iv) $\kappa\left(\mathcal{C}_{g}^{1}, \hat{\xi}\right)$ freely generates $H_{\text {êt }}^{1}\left(\mathcal{M}_{g}^{1} \otimes \bar{K}, \mathbb{H}_{\mathbb{Z}_{\ell}}\right)$.

Moreover, $\tilde{\nu}^{\text {an }}\left(\mathcal{C}_{g}^{1}, \hat{\xi}\right)$ and $\tilde{\nu}\left(\mathcal{C}_{g}^{1}, \hat{\xi}\right)$ correspond under the comparison isomorphism

$$
H^{1}\left(\mathcal{M}_{g}^{1} \text { an }, \mathbb{L}_{\mathbb{Z}} / \mathbb{H}_{\mathbb{Z}}\right) \otimes \mathbb{Z}_{\ell} \cong H_{\text {ét }}^{1}\left(\mathcal{M}_{g}^{1} \otimes \bar{K}, \mathbb{L}_{\mathbb{Z}_{\ell}} / \mathbb{H}_{\mathbb{Z}_{\ell}}\right)
$$

and $\kappa^{\text {an }}\left(\mathcal{C}_{g}^{1}, \hat{\xi}\right)$ and $\kappa\left(\mathcal{C}_{g}^{1}, \hat{\xi}\right)$ correspond under the comparison isomorphism

$$
H^{1}\left(\mathcal{M}_{g}^{1 \text { an }}, \mathbb{H}_{\mathbb{Z}}\right) \otimes \mathbb{Z}_{\ell} \cong H_{\text {ét }}^{1}\left(\mathcal{M}_{g}^{1} \otimes \bar{K}, \mathbb{H}_{\mathbb{Z}_{\ell}}\right)
$$

\footnotetext{
${ }^{2}$ Constructed explicitly in 9 .
} 
Proof. It suffices to prove the analytical version, as the étale version then follows via comparison theorems. We therefore restrict ourselves to the analytical case. By Corollary 6.3 both groups are torsion free groups of rank one. So it suffices to show that these classes are both non-zero and are not divisible by a positive integer.

By Proposition 6.4 to prove (i), it suffices to show that the image of $\hat{m}^{\text {an }}$ in $H^{1}\left(\Gamma_{S, x}, L_{\mathbb{Z}} / H_{\mathbb{Z}}\right)$ is not divisible. But this is true because the image of $\hat{m}^{\text {an }}$ under the inclusion

$$
H^{1}\left(\Gamma_{S, x}, L_{\mathbb{Z}} / H_{\mathbb{Z}}\right) \hookrightarrow \operatorname{Hom}_{\mathrm{Sp}\left(H_{\mathbb{Z}}\right)}\left(H_{1}\left(T_{S, x}\right), L_{\mathbb{Z}} / H_{\mathbb{Z}}\right)
$$

is $2 \tau_{S, x}$, and because Morita's computations [27, p. 220] show that $\tau_{S, x}$ is not in the image of this map.

We now prove that $\kappa^{\text {an }}\left(\mathcal{C}_{g}^{1}, \hat{\xi}\right)$ is not divisible. For convenience, we denote it by $\kappa^{\text {an }}$. We have surjections

$$
H_{1}\left(T_{S, x}\right) \rightarrow L_{\mathbb{Z}} \rightarrow H_{\mathbb{Z}}
$$

By [29] Cor. 6.7], the image of $\mu^{\text {an }}$ under the induced mapping $H^{1}\left(\mathcal{M}_{g}^{1 \text { an }}, \mathbb{L}_{\mathbb{Z}}\right) \rightarrow$ $H^{1}\left(\mathcal{M}_{g}^{1}\right.$ an, $\left.\mathbb{H}_{\mathbb{Z}}\right)$ is $\kappa^{\text {an }}$. Since the above mapping is surjective, and since $\mu^{\text {an }}$ goes to $2 \tau_{S, x}$, it follows that if $\kappa^{\text {an }} / d$ is integral and $d>1$, then $d=2$. But this is impossible as it would imply that the section $(2 g-2) x-K_{C}$ of the relative jacobian $\mathcal{J} \rightarrow \mathcal{M}_{g}^{1}$ an is divisible by 2 , which would imply that there is a square root of $K_{C}$ defined over $\mathcal{M}_{g}^{1}$. If $-2 \sigma=(2 g-2) x-K_{C}$, then

$$
K_{C}=2(\sigma+(g-1) x) .
$$

That is, there is a theta characteristic defined over $\mathcal{M}_{g}^{1 \text { an }}$. But there is no such theta characteristic. It follows that $\kappa^{\text {an }}$ generates $H^{1}\left(\mathcal{M}_{g}^{1}\right.$ an, $\left.\mathbb{H}_{Z}\right)$.

The statement that $\kappa^{\text {an }}$ is not divisible also follows from Pulte's result and 26, Prop. 6.4].

The wedging with the symplectic element map induces a homomorphism

$$
q_{*}: H^{1}\left(\mathcal{M}_{g}^{1 \text { an }}, \mathbb{H}_{\mathbb{Z}}\right) \rightarrow H^{1}\left(\mathcal{M}_{g}^{1} \text { an }, \mathbb{L}_{\mathbb{Z}}\right) .
$$

Likewise in the étale case:

Corollary 6.6. If $g \geq 3$, then

$$
H^{1}\left(\mathcal{M}_{g}^{1 \text { an }}, \mathbb{L}_{\mathbb{Z}}\right)=\mathbb{Z} q_{*} \kappa^{\text {an }}\left(\mathcal{C}_{g}^{1}, \hat{\xi}\right) \oplus \mathbb{Z} \mu^{\text {an }}\left(\mathcal{C}_{g}^{1}, \hat{\xi}\right) .
$$

Proof. This follows immediately from the previous result using the exact sequence of cohomology groups

$$
\cdots \rightarrow H^{1}\left(\mathcal{M}_{g}^{1 \text { an }}, \mathbb{H}_{\mathbb{Z}}\right) \rightarrow H^{1}\left(\mathcal{M}_{g}^{1 \text { an }}, \mathbb{L}_{\mathbb{Z}}\right) \rightarrow H^{1}\left(\mathcal{M}_{g}^{1 \text { an }}, \mathbb{L}_{\mathbb{Z}} / \mathbb{H}_{\mathbb{Z}}\right) \rightarrow \cdots
$$

associated to the short exact sequence $0 \rightarrow \mathbb{H}_{\mathbb{Z}} \stackrel{q}{\rightarrow} \mathbb{L}_{\mathbb{Z}} \rightarrow \mathbb{L}_{\mathbb{Z}} / \mathbb{H}_{\mathbb{Z}} \rightarrow 0$ of coefficients.

Corollary 6.7. If $g \geq 3$, the natural homomorphism

$$
H_{\text {ét }}^{1}\left(\mathcal{M}_{g}^{1}, \mathbb{L}_{\mathbb{Z}_{\ell}}\right) \rightarrow H_{\text {ét }}^{1}\left(\mathcal{M}_{g}^{1} \otimes \bar{K}, \mathbb{L}_{\mathbb{Z}_{\ell}}\right)
$$

is an isomorphism. Moreover, both groups are generated freely by $\mu\left(\mathcal{C}_{g}^{1}, \hat{\xi}\right)$ and $q_{*} \kappa\left(\mathcal{C}_{g}^{1}, \hat{\xi}\right)$. 
Proof. The fact $H_{\text {ét }}^{1}\left(\mathcal{M}_{g}^{1} \otimes \bar{K}, \mathbb{L}_{\mathbb{Z}_{\ell}}\right)$ is freely generated by $\mu\left(\mathcal{C}_{g}^{1}, \hat{\xi}\right)$ and $q_{*} \kappa\left(\mathcal{C}_{g}^{1}, \hat{\xi}\right)$ follows from the previous result via comparison theorems. Since these classes are restrictions of classes in $H_{\text {ét }}^{1}\left(\mathcal{M}_{g}^{1}, \mathbb{L}_{\mathbb{Z}_{\ell}}\right)$, they are Galois invariant. Using the spectral sequence $^{3}$

$$
H^{s}\left(G_{K}, H_{\text {ét }}^{t}\left(\mathcal{M}_{g}^{1} \otimes \bar{K}, \mathbb{L}_{\mathbb{Z}_{\ell}}\right)\right) \Longrightarrow H_{\text {ét }}^{s+t}\left(\mathcal{M}_{g}^{1}, \mathbb{L}_{\mathbb{Z}_{\ell}}\right) .
$$

constructed in [16] and the fact that $H^{0}\left(\mathcal{M}_{g}^{1} \otimes \bar{K}, \mathbb{L}_{\mathbb{Z}_{\ell}}\right)$ vanishes, which follows directly from Proposition [5.8 it follows that

$$
\begin{aligned}
H_{\text {èt }}^{1}\left(\mathcal{M}_{g}^{1}, \mathbb{L}_{\mathbb{Z}_{\ell}}\right) \cong H^{0}\left(G_{K}, H_{\text {ét }}^{1}\left(\mathcal{M}_{g}^{1} \otimes \bar{K}, \mathbb{L}_{\mathbb{Z}_{\ell}}\right)\right) & \\
& \cong H_{\text {ét }}^{1}\left(\mathcal{M}_{g}^{1} \otimes \bar{K}, \mathbb{L}_{\mathbb{Z}_{\ell}}\right) \cong \mathbb{Z}_{\ell} q_{*} \kappa\left(\mathcal{C}_{g}^{1}, \hat{\xi}\right) \oplus \mathbb{Z}_{\ell} \mu\left(\mathcal{C}_{g}^{1}, \hat{\xi}\right) .
\end{aligned}
$$

Remark 6.8. It is worth noting that the image of $\mu^{\text {an }}\left(\mathcal{C}_{g}^{1}, \hat{\xi}\right)$ under the homomorphism

$$
H^{1}\left(\mathcal{M}_{g}^{1 \text { an }}, \mathbb{L}_{\mathbb{Z}}\right) \rightarrow H^{1}\left(\mathcal{M}_{g}^{1 \text { an }}, \mathbb{H}_{\mathbb{Z}}\right)
$$

induced by the contraction $c: \mathbb{L}_{\mathbb{Z}} \rightarrow \mathbb{H}_{\mathbb{Z}}$, defined in Section 5.4 is $\kappa^{\text {an }}\left(\mathcal{C}_{g}^{1}, \hat{\xi}\right)$. A proof can be found in [29, Cor. 6.7]. It thus follows from comparison theorems that the image of $\mu\left(\mathcal{C}_{g}^{1}, \hat{\xi}\right)$ under the map

$$
H_{\text {ét }}^{1}\left(\mathcal{M}_{g}^{1}, \mathbb{L}_{\mathbb{Z}_{\ell}}\right) \rightarrow H_{\text {ét }}^{1}\left(\mathcal{M}_{g}^{1}, \mathbb{H}_{\mathbb{Z}_{\ell}}\right)
$$

induced by the contraction $c$ is $\kappa\left(\mathcal{C}_{g}^{1}, \hat{\xi}\right)$.

For cohomology with $\mathbb{Q}_{\ell}$-module coefficients, the results of this section may be summarized by the following easily proved extension of Proposition 6.2

Theorem 6.9. If $g \geq 3$ and $V$ is a finite dimensional irreducible representation of $\operatorname{GSp}\left(H_{\mathbb{Q}_{\ell}}\right)$, then $H_{\text {ét }}^{1}\left(\mathcal{M}_{g}^{1}, \mathbb{V}\right)$ vanishes unless $V$ is isomorphic to $H_{\mathbb{Q}_{\ell}}, L_{\mathbb{Q}_{\ell}} / H_{\mathbb{Q}_{\ell}}$ or some $\mathbb{Q}_{\ell}(n)$. In these cases we have

$$
H_{\text {ét }}^{1}\left(\mathcal{M}_{g}^{1}, \mathbb{H}_{\mathbb{Q}_{\ell}}\right) \cong \mathbb{Q}_{\ell} \kappa\left(\mathcal{C}_{g}^{1}, \hat{\xi}\right) \text { and } H_{\text {ét }}^{1}\left(\mathcal{M}_{g}^{1}, \mathbb{L}_{\mathbb{Q}_{\ell}} / \mathbb{H}_{\mathbb{Q}_{\ell}}\right) \cong \mathbb{Q}_{\ell} \nu\left(\mathcal{C}_{g}^{1}\right)
$$

and $H_{\text {êt }}^{1}\left(\mathcal{M}_{g}^{1}, \mathbb{Q}_{\ell}(n)\right) \cong H^{1}\left(G_{K}, \mathbb{Q}_{\ell}(n)\right)$.

\section{Proof of the Density Theorems}

We begin by relating the notation of the Introduction to that of the preceding sections. Throughout the remainder of this paper, $K$ is a subfield of $\mathbb{C}$ and $(C, \xi)$ is a pointed smooth projective curve over $K$ of genus $g \geq 3$. As in the introduction, we denote the $\mathbb{Q}_{\ell}$-Lie algebra of $\pi_{1}^{\text {un }}\left(C^{\text {an }}, \xi^{\text {an }}\right)$ by $\mathfrak{p}(C, \xi)$. Since the exponential mapping $\mathfrak{p}(C, \xi) \rightarrow \pi_{1}^{\text {un }}(C, \xi)$ is an isomorphism of provarieties, and since, this is an isomorphism of prounipotent groups if we endow $\mathfrak{p}(C, \xi)$ with the product given by the Baker-Campbell-Hausdorff formula, the exponential mapping induces a natural group isomorphism

$$
\operatorname{Aut} \mathfrak{p}(C, \xi) \stackrel{\simeq}{\longrightarrow} \operatorname{Aut} \pi_{1}^{\text {un }}\left(C^{\text {an }}, \xi^{\text {an }}\right) .
$$

\footnotetext{
${ }^{3}$ Note that this spectral sequence uses the definition of étale cohomology given in 16. Although the definitions of $H_{\text {ét }}^{\bullet}$ in [16] and [25] differ in general, they coincide for $H_{\text {ét }}^{1}$.
} 
The homomorphisms $\rho_{C, \xi}$ and $\theta_{C, \xi}$ defined in the Introduction fit into the commutative diagram

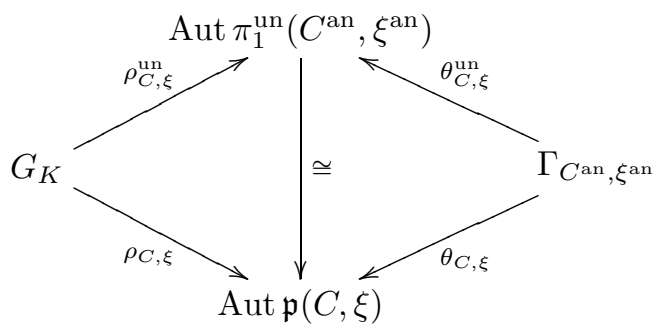

In short, we have removed the decoration "un" when the target is $\operatorname{Aut} \mathfrak{p}(C, \xi)$ after making the natural identifications.

Since $K$ is embedded in $\mathbb{C}$, we can naturally regard $[C, \xi]$ as a $\mathbb{C}$-rational point of $\mathcal{M}_{g}^{1}$ to which corresponds the homomorphism

$$
\hat{\rho}_{C, \xi}: \pi_{1}^{\text {alg }}\left(\mathcal{M}_{g}^{1},[C, \xi]\right) \rightarrow \operatorname{Aut} \mathfrak{p}(C, \xi)
$$

that extends both $\theta_{C, \xi}$ and $\rho_{C, \xi}$ :

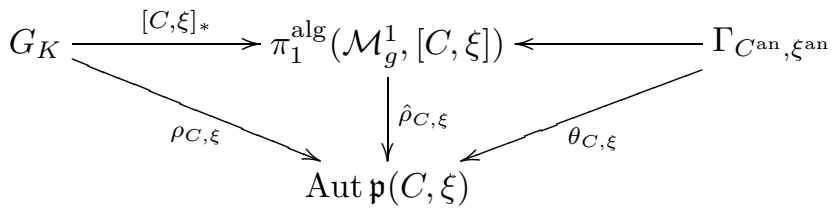

7.1. Strategy. We shall prove each of the 3 statements of Theorem 1 together with the following:

(iv) The Zariski closure of the image of $\rho_{C, \xi}$ in $\operatorname{Aut} \mathfrak{p}(C, \xi)$ is identical with that of the image of $\hat{\rho}_{C, \xi}$.

We shall do this by proving that (i) $\Rightarrow$ (ii) $\Rightarrow$ (iii) $\Rightarrow$ (iv) $\Rightarrow$ (i). The implications (iv) $\Rightarrow$ (i) $\Rightarrow$ (ii) are obvious. In this section, we shall prove (ii) $\Rightarrow$ (iii) and (iii) $\Rightarrow$ (iv) to complete the proof.

7.2. Proof of (ii) $\Rightarrow$ (iii). First, if the closure of im $\rho_{C, \xi}^{2}$ contains $\operatorname{im} \theta_{C, \xi}^{2}$, then the closure of the image of $\rho_{C, \xi}^{1}$ contains $\operatorname{im} \theta_{C, \xi}^{1}$. The image of $\theta_{C, \xi}^{1}$ is $\operatorname{Sp}\left(H_{\mathbb{Z}}\right)$, and therefore has Zariski closure $\operatorname{Sp}\left(H_{\mathbb{Q}_{\ell}}\right)$ in $\operatorname{GSp}\left(H_{\mathbb{Q}_{\ell}}\right)$. Since the composite

$$
G_{K} \stackrel{\rho_{7}}{\rightarrow} \operatorname{GSp}\left(H_{\mathbb{Q}_{\ell}}\right) \stackrel{\chi}{\rightarrow} \mathbb{G}_{m}
$$

is the $\ell$-adic cyclotomic character, and since it is assumed to have infinite image, it follows that if the Zariski closure of im $\rho_{C, \xi}^{1}$ contains $\operatorname{im} \theta_{C, \xi}^{1}$, then its Zariski closure is all of $\operatorname{GSp}\left(H_{\mathbb{Q}_{\ell}}\right)$.

It is well-known and easy to show that the Lie algebra $\mathfrak{p}(C, \xi) / L^{3}$ is (unnaturally) isomorphic to the graded Lie algebra

$$
H_{\mathbb{Q}_{\ell}} \oplus \Lambda^{2} H_{\mathbb{Q}_{\ell}} / q
$$

where $q$ spans the $\operatorname{Sp}\left(\mathbb{H}_{\mathbb{Q}_{\ell}}\right)$ invariants in $\Lambda^{2} H_{\mathbb{Q}_{\ell}}$. The bracket is given by the mapping

$$
H_{\mathbb{Q}_{\ell}} \otimes H_{\mathbb{Q}_{\ell}} \rightarrow \Lambda^{2} H_{\mathbb{Q}_{\ell}} / q
$$


that takes $a \otimes b$ to $a \wedge b \bmod q$. Using this description, it is easy to see that Aut $\left[\mathfrak{p}(C, \xi) / L^{3}\right]$ is an extension

$$
0 \rightarrow \operatorname{Hom}\left(H_{\mathbb{Q}_{\ell}}, \Lambda^{2} H_{\mathbb{Q}_{\ell}} / q\right) \rightarrow \operatorname{Aut}\left[\mathfrak{p}(C, \xi) / L^{3}\right] \rightarrow \operatorname{GSp}\left(H_{\mathbb{Q}_{\ell}}\right) \rightarrow 1
$$

The kernel is abelian. When $g \geq 3$, the kernel decomposes as an GSp-module into three irreducible components:

$$
\operatorname{Hom}\left(H_{\mathbb{Q}_{\ell}}, \Lambda^{2} H_{\mathbb{Q}_{\ell}} / q\right) \cong H_{\mathbb{Q}_{\ell}} \oplus L_{\mathbb{Q}_{\ell}} / H_{\mathbb{Q}_{\ell}} \oplus U(-1)
$$

where $U$ is the irreducible representation corresponding to the partition $[2,1] .{ }^{4}$ Each component has weight -1 as a $\operatorname{GSp}\left(H_{\mathbb{Q}_{\ell}}\right)$-module.

To proceed, we need to recall an elementary fact about group cohomology. Suppose that $R$ is a reductive affine algebraic group over $\mathbb{Q}_{\ell}$ and that $V$ is an irreducible $R$-module. Suppose $\Gamma$ is a profinite group and that $r: \Gamma \rightarrow R\left(\mathbb{Q}_{\ell}\right)$ is a continuous Zariski dense representation. Each lift of $r$ to a homomorphism $\tilde{r}: \Gamma \rightarrow R \ltimes V$, determines a cohomology class

$$
c \in H^{1}(\Gamma, V)
$$

by taking the class of the cocycle $f$ obtained by taking the second component of $\tilde{r}$ :

$$
\tilde{r}(g)=(r(g), f(g)) \in R \ltimes V .
$$

The class $c$ determines $\tilde{r}$ up to conjugation by an element of $V$. The following result is easily proved. Details are left to the reader.

Lemma 7.1. The Zariski closure of the image of the lift $\tilde{r}$ contains $V$ if and only if $c$ is not zero.

Set

$$
E=\operatorname{Hom}\left(H_{\mathbb{Q}_{\ell}},\left[\Lambda^{2} H_{\mathbb{Q}_{\ell}}\right] / q\right) .
$$

This decomposes as the sum of one copy each of $V_{\lambda}$ for $\lambda \in\left\{[1],\left[1^{3}\right](-1),[2,1](-1)\right\}$. The group Aut $\left[\mathfrak{p}(C, \xi) / L^{3}\right]$ is an extension

$$
0 \rightarrow E \rightarrow \text { Aut }\left[\mathfrak{p}(C, \xi) / L^{3}\right] \rightarrow \operatorname{GSp}\left(H_{\mathbb{Q}_{\ell}}\right) \rightarrow 1
$$

Since the diagram

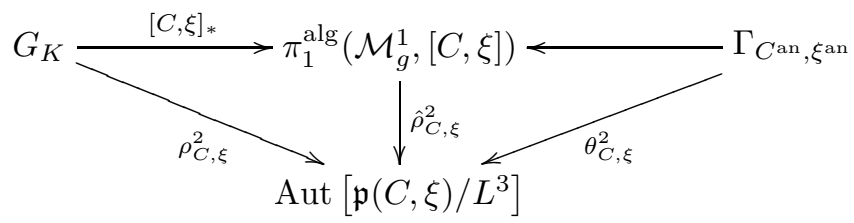

commutes, the construction above gives characteristic classes

$$
c_{\lambda} \in H^{1}\left(G_{K}, E\right), \tilde{c}_{\lambda} \in H^{1}\left(\pi_{1}^{\mathrm{alg}}\left(\mathcal{M}_{g}^{1},[C, \xi]\right), E\right), \tilde{c}_{\lambda}^{\mathrm{an}} \in H^{1}\left(\Gamma_{C^{\mathrm{an}}, \xi^{\mathrm{an}}}, E\right)
$$

for each $\lambda \in\left\{[1],\left[1^{3}\right](-1),[2,1](-1)\right\}$. Note that $\tilde{c}_{\lambda}$ maps to $c_{\lambda}$ and $\tilde{c}_{\lambda}^{\text {an }}$ under the induced maps

$$
H^{1}\left(G_{K}, E\right) \leftarrow H^{1}\left(\pi_{1}^{\text {alg }}\left(\mathcal{M}_{g}^{1},[C, \xi]\right), E\right) \rightarrow H^{1}\left(\Gamma_{C^{\text {an }}, \xi^{\text {an }}}, E\right) .
$$

\footnotetext{
${ }^{4}$ Each partition $\lambda$ of a non-negative integer $m$ into $\leq g$ pieces corresponds to an irreducible representation of $\operatorname{Sp}(H)$. Each of these can be lifted uniquely to a representation $V_{\lambda}$ of $\mathrm{GSp}(H)$ where the scalar matrix $a I$ acts as $a^{m} \operatorname{id}_{V}$. Every other representation of $\operatorname{GSp}(H)$ can be obtained from such a $V_{\lambda}$ by tensoring with the one dimensional representation $\mathbb{Z}(n)$ for some $n$. We shall denote the weight of $V_{\lambda}(n):=V_{\lambda} \otimes \mathbb{Z}(n)$ by $\lambda(n)$. This has weight $-m-2 n$, which means that the scalar matrix $a I$ acts as $a^{m+2 n} \operatorname{id}_{V}$ on it.
} 
It follows from [10, Cor. 9.2] that the Zariski closure of the image of $\theta_{C, \xi}^{2}$ contains $V_{[1]}$ and $V_{\left[1^{3}\right](-1)}$, but does not intersect $V_{[2,1](-1)}$. It follows that the Zariski closure of $\rho_{C, \xi}^{2}$ contains $\operatorname{im} \theta_{C, \xi}^{2}$ if and only if $c_{[1]}$ and $c_{\left[1^{3}\right](-1)}$ are both non-zero. To complete the proof that Proof of (ii) $\Rightarrow$ (iii) we will show that $c_{[1]}$ is a non-zero multiple of $\kappa(C, \xi)$ and $c_{\left[1^{3}\right](-1)}$ is a non-zero multiple of $\nu(C)$.

To prove this, note that [10, Cor. 9.2] implies that $\tilde{C}_{[1]}^{\text {an }}$ and $\tilde{c}_{\left[1^{3}\right](-1)}^{\text {an }}$ are both nonzero and therefore, by Proposition [6.5] $\tilde{c}_{[1]}^{\text {an }}$ is a non-zero multiple of $\kappa^{\text {an }}\left(\mathcal{C}_{g}^{1}, \hat{\xi}\right)$ and $\tilde{c}_{\left[1^{3}\right](-1)}^{\text {an }}$ is a non-zero multiple of $\tilde{\nu}^{\text {an }}\left(\mathcal{C}_{g}^{1}, \hat{\xi}\right)$. Another application of Proposition 6.5 implies that $\tilde{c}_{[1]}$ is a non-zero multiple of $\kappa\left(\mathcal{C}_{g}^{1}, \hat{\xi}\right)$ and that $\tilde{c}_{\left[1^{3}\right](-1)}$ is a non-zero multiple of $\tilde{\nu}\left(\mathcal{C}_{g}^{1}, \hat{\xi}\right)$. Propositions 4.3 and 4.5 now imply that $c_{[1]}$ is a non-zero multiple of $\kappa(C, \xi)$ and that $c_{\left[1^{3}\right](-1)}$ is a non-zero multiple of $\nu(C)=\tilde{\nu}(C, \xi)$, as claimed.

7.3. Weighted completion. To prove (iii) $\Rightarrow$ (iv) we will need the tool of weighted completion.

We begin with a brief review of the theory of weighted completion of a profinite group. More details (including complete proofs) can be found in [11] and [12].

Suppose that $F$ is a topological field, $R$ a reductive affine algebraic group defined over $F$, that $\Gamma$ is a profinite group, and that $r: \Gamma \rightarrow R(F)$ is a continuous homomorphism with Zariski dense image. In addition, we fix a central cocharacter $w: \mathbb{G}_{m} \rightarrow R$, that is, a homomorphism from $\mathbb{G}_{m}$ whose image lies in the center of $R$.

A $\Gamma$-module is a finite dimensional $F$-vector space on which $\Gamma$ acts continuously. Let $F(m)$ denote a one-dimensional $F$-vector space on which $\mathbb{G}_{m}$ acts by $m$-th power multiplication. An $R$-module is of pure weight $m$ if it is isomorphic to a sum of copies of $F(m)$ as a $\mathbb{G}_{m}$-module via $w$. Schur's lemma implies that each irreducible $R$-module is pure of weight $m$ for some integer $m$.

A weighted $\Gamma$-module is a finite dimensional $\Gamma$-module $M$ with an increasing filtration (necessarily unique)

$$
0=W_{n} M \subseteq W_{n+1} M \subseteq \cdots \subseteq W_{N-1} M \subseteq W_{N} M=M,
$$

by $\Gamma$-submodules, where, for each integer $m$, the weight graded quotient $\operatorname{Gr}_{m}^{W} M:=$ $W_{m} M / W_{m-1} M$ is an $R$-module of weight $m$ in such a way that the action of $\Gamma$ on it factors through this $R$ action:

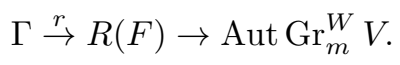

One fact that makes weighted $\Gamma$-modules useful is:

Proposition 7.2 (11]). The functor $\mathrm{Gr}_{\bullet}^{W}$ is exact on the category of weighted Г-modules.

The category of weighted $\Gamma$-modules is tannakian with fiber functor the functor that takes a weighted $\Gamma$-module to its underlying vector space.

The weighted completion of $\Gamma$ with respect to $r$ and $\omega$ is the fundamental group of the tannakian category of weighted $\Gamma$-modules with respect to the fiber functor above. We shall denote it by $\mathcal{G}(\Gamma, r)$. It is a pro-algebraic group over $F$, which is an extension

$$
1 \rightarrow \mathcal{U}(\Gamma, r) \rightarrow \mathcal{G}(\Gamma, r) \rightarrow R \rightarrow 1
$$


of $R$ by a prounipotent group $\mathcal{U}(\Gamma, r)$. There is a natural homomorphism $\tilde{r}: \Gamma \rightarrow$ $\mathcal{G}(\Gamma, r)(F)$ that lifts $r$, and is easily seen to be Zariski dense. The action of $\Gamma$ on a weighted $\Gamma$-module factors through this homomorphism.

The setting. Fix a pointed smooth projective curve $(C, \xi)$ of genus $\geq 3$ over the subfield $K$ of $\mathbb{C}$. Denote the dual of $H_{\text {ét }}^{1}\left(C \otimes \bar{K}, \mathbb{Q}_{\ell}\right)$ by $H_{\mathbb{Q}_{\ell}}$. In the rest of this section, $F$ will be $\mathbb{Q}_{\ell}$ and $R$ will be $\operatorname{GSp}\left(H_{\mathbb{Q}_{\ell}}\right)$. The central cocharacter is the one that takes $a \in \mathbb{G}_{m}$ to $a^{-1}$ id. The group $\Gamma$ is any that acts naturally on $C$, such as $G_{K}$ or $\pi_{1}^{\text {alg }}\left(\mathcal{M}_{g}^{1},\left[C_{x}, x\right]\right)$, where $x$ is a geometric point of $C$ lying over $\xi$. We suppose that the induced representation $r: \Gamma \rightarrow \operatorname{GSp}\left(H_{\mathbb{Q}_{\ell}}\right)$ is Zariski dense. We thus have the weighed completion $\mathcal{G}(\Gamma, r)$.

Proposition 7.3. In this setting, $\mathfrak{p}(C, \xi)$ is the inverse limit of weighted $\Gamma$-modules. The action $\Gamma \rightarrow \operatorname{Aut} \mathfrak{p}(C, \xi)$ thus induces a homomorphism $\mathcal{G}(\Gamma, r) \rightarrow \operatorname{Aut} \mathfrak{p}(C, \xi)$.

Proof. Define a weight filtration on $\mathfrak{p}(C, \xi)$ by by

$$
W_{-m} \mathfrak{p}(C, \xi)=L^{m} \mathfrak{p}(C, \xi) .
$$

Since the lower central series of $\mathfrak{p}(C, \xi)$ is characteristic, it is preserved by $\Gamma$. Since $H_{\mathbb{Q}_{\ell}}$ has weight -1 , and since the iterated bracket mapping

$$
H_{\mathbb{Q} \ell}^{\otimes m} \rightarrow \mathrm{Gr}_{-m}^{W} \mathfrak{p}(C, \xi), \quad x_{1} \otimes \cdots \otimes x_{m} \mapsto\left[x_{1}\left[x_{2}\left[\cdots\left[x_{m-1}, x_{m}\right]\right] \cdots\right]\right]
$$

is surjective and $\Gamma$-invariant, it follows that $\operatorname{Gr}_{-m}^{W} \mathfrak{p}(C, \xi)$ is a $\operatorname{GSp}\left(H_{\mathbb{Q}_{\ell}}\right)$-module of weight $-m$ and that each $\mathfrak{p}(C, \xi)$ is the inverse limit of the weighted $\Gamma$-modules $\mathfrak{p}(C, \xi) / W_{-m}$.

We conclude this paragraph with a surjectivity criterion which will be used in the proof of (iii) $\Rightarrow$ (iv).

Let $F$ be a topological field and $\Gamma_{j}(j=1,2)$ be two topological groups. Suppose that $R$ is a reductive group over $F$ with central cocharacter $w: \mathbb{G}_{m} \rightarrow R$ as above. Suppose also that we have two Zariski dense representations $r_{j}: \Gamma_{j} \rightarrow R(F)$. We then have the two corresponding weighted completions by

$$
\tilde{\rho}_{j}: \Gamma_{j} \rightarrow \mathcal{G}\left(\Gamma_{j}\right) \quad j=1,2 .
$$

A homomorphism $\phi: \Gamma_{1} \rightarrow \Gamma_{2}$ that commutes with the projections to $R(F)$ induces a homomorphism $\phi_{\mathcal{G}}: \mathcal{G}_{1} \rightarrow \mathcal{G}_{2}$ that commutes with the projections to $R$.

Lemma 7.4. The homomorphism $\phi_{\mathcal{G}}: \mathcal{G}\left(\Gamma_{1}\right) \rightarrow \mathcal{G}\left(\Gamma_{2}\right)$ is surjective if and only if the induced mappings

$$
H^{1}\left(\Gamma_{2}, V_{\alpha}\right) \rightarrow H^{1}\left(\Gamma_{1}, V_{\alpha}\right)
$$

are injective for all negatively weighted irreducible representation $V_{\alpha}$ of $R$.

Proof. Denote the prounipotent radical of $\mathcal{G}\left(\Gamma_{j}\right)$ by $\mathcal{U}_{j}, j=1,2$. Since the two $\mathcal{G}\left(\Gamma_{j}\right)$ have the same reductive quotient, $\phi_{\mathcal{G}}$ is surjective if and only if its restriction

$$
\phi_{\mathcal{U}}: \mathcal{U}_{1} \rightarrow \mathcal{U}_{2}
$$

is surjective. This occurs if and only if the induced mapping $d \phi_{\mathcal{U}}: \mathfrak{u}_{1} \rightarrow \mathfrak{u}_{2}$ on Lie algebras is surjective. Since the $\mathfrak{u}_{j}$ are pronilpotent, this occurs if and only if the induced mapping

$$
\phi_{\mathcal{U}, *}: H_{1}\left(\mathfrak{u}_{1}\right) \rightarrow H_{1}\left(\mathfrak{u}_{2}\right)
$$


on abelianizations is surjective. The $H_{1}\left(\mathfrak{u}_{j}\right)$ are weighted modules and $\phi_{\mathfrak{U}, *}$ preserves the weight filtration (cf. [11, $\S 3.3,3.4]$ ). By the exactness of $\operatorname{Gr}_{\bullet}^{W}, \phi_{\mathcal{U}, *}$ is surjective, if and only if

$$
\mathrm{Gr}_{\bullet}^{W} \phi \mathcal{U}, *: \mathrm{Gr}_{\bullet}^{W} H_{1}\left(\mathfrak{u}_{1}\right) \rightarrow \mathrm{Gr}_{\bullet}^{W} H_{1}\left(\mathfrak{u}_{2}\right)
$$

is surjective. Dualizing, this is true if and only if

$$
\phi_{\mathcal{U}}^{*}: H^{1}\left(\mathrm{Gr}_{\bullet}^{W} \mathfrak{u}_{2}\right) \rightarrow H^{1}\left(\mathrm{Gr}_{\bullet}^{W} \mathfrak{u}_{1}\right)
$$

is injective. By [11, Thm. 4.8], there are natural isomorphisms

$$
H^{1}\left(\mathrm{Gr}_{\bullet}^{W} \mathfrak{u}_{j}\right) \cong \bigoplus_{\alpha} H^{1}\left(\Gamma_{j}, V_{\alpha}\right) \otimes V_{\alpha}^{*}
$$

where $\left(V_{\alpha}\right)_{\alpha}$ is a set of representatives of the isomorphism classes of negatively weighted irreducible representations of $R$. The result follows as the mapping (17) is induced by the mappings

$$
\phi^{*}: H^{1}\left(\Gamma_{2}, V_{\alpha}\right) \rightarrow H^{1}\left(\Gamma_{1}, V_{\alpha}\right) .
$$

7.4. Proof of (iii) $\Rightarrow$ (iv). Assume the conditions of (iii). Denote the weighted completion of $G_{K}$ with respect to

$$
\rho_{C, \xi}^{1}: G_{K} \rightarrow \operatorname{GSp}\left(H_{\mathbb{Q}_{\ell}}\right)
$$

by $\mathcal{G}\left(G_{K}\right)$, and the weighted completion of $\pi_{1}^{\text {alg }}\left(\mathcal{M}_{g}^{1},[C, \xi]\right)$ with respect to

$$
\hat{\rho}_{C, \xi}^{1}: \pi_{1}^{\operatorname{alg}}\left(\mathcal{M}_{g}^{1},[C, \xi]\right) \rightarrow \operatorname{GSp}\left(H_{\mathbb{Q}_{\ell}}\right)
$$

by $\mathcal{G}\left(\mathcal{M}_{g}^{1}\right)$.

Since the actions

$$
\rho_{C, \xi}: G_{K} \rightarrow \operatorname{Aut} \mathfrak{p}(C, \xi) \text { and } \rho_{\mathcal{C}_{g}^{1}, \hat{\xi}}: \pi_{1}^{\operatorname{alg}}\left(\mathcal{M}_{g}^{1},\left[C_{x}, \tilde{x}\right]\right) \rightarrow \operatorname{Aut} \mathfrak{p}(C, \xi)
$$

are both negatively weighted, they induce representations

$$
\mathcal{G}\left(G_{K}\right) \longrightarrow \operatorname{Aut} \mathfrak{p}(C, \xi) \longleftarrow \mathcal{G}\left(\mathcal{M}_{g}^{1}\right) .
$$

The homomorphism

$$
[C, \xi]_{*}: G_{K} \rightarrow \pi_{1}^{\mathrm{alg}}\left(\mathcal{M}_{g}^{1},[C, \xi]\right)
$$

commutes with the projections to $\operatorname{GSp}\left(H_{\mathbb{Q}_{\ell}}\right)$, and thus induces a homomorphism

$$
(C, \xi)_{*}: \mathcal{G}\left(G_{K}\right) \rightarrow \mathcal{G}\left(\mathcal{M}_{g}^{1}\right) \text {. }
$$

The commutativity of the left-hand diagram below implies the commutativity of the right-hand one:
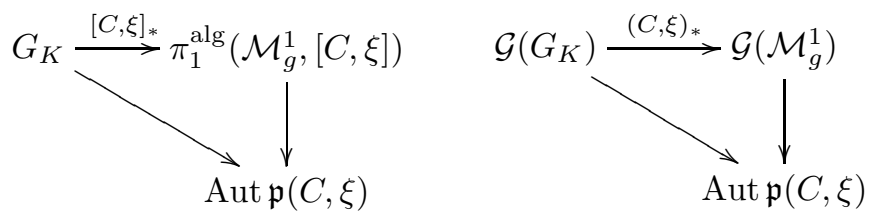

Since the natural homomorphism $G_{K} \rightarrow \mathcal{G}\left(G_{K}\right)$ and $\pi_{1}^{\text {alg }}\left(\mathcal{M}_{g}^{1},[C, \xi]\right) \rightarrow \mathcal{G}\left(\mathcal{M}_{g}^{1}\right)$ have Zariski dense image and, given the commutativity of (16), (iii) implies (iv) follows directly from the following more general result. 
Theorem 7.5. Assuming that the image of $\rho_{C}^{1}: G_{K} \rightarrow \mathrm{GSp}\left(H_{\mathbb{Q}_{\ell}}\right)$ is Zariski dense, the homomorphism $(C, \xi)_{*}$ is surjective if and only if the classes $\nu(C)$ and $\kappa(C, \xi)$ both have infinite order.

Proof. This follows directly from Proposition 4.5 Lemma[7.4 and Theorem 6.9

\section{The $\ell$-Adic Harris-Pulte Theorem}

In this section we prove the $\ell$-adic Harris-Pulte Theorem. We do this by proving a more general statement, and deducing it by specialization.

A pointed curve $(C, \xi)$ over $K$ and a geometric point $x$ of Spec $K$ gives rise to a homomorphism

$$
\delta_{C, x}: \pi_{1}^{\mathrm{alg}}\left(\mathcal{M}_{g}^{1},\left[C_{x}, \tilde{x}\right]\right) \rightarrow \operatorname{Aut} \pi_{1}^{(\ell)}\left(C_{x}, \tilde{x}\right),
$$

where $\tilde{x}=\xi(x)$, and therefore a class

$$
\delta_{C, x}^{*} \hat{m}^{(\ell)} \in H^{1}\left(\pi_{1}^{\operatorname{alg}}\left(\mathcal{M}_{g}^{1},\left[C_{x}, \tilde{x}\right]\right), L_{\mathbb{Z}_{\ell}}\right) \cong H_{\text {ét }}^{1}\left(\mathcal{M}_{g}^{1}, \mathbb{L}_{\mathbb{Z}_{\ell}}\right),
$$

where $\hat{m}^{(\ell)}$ is the class defined in $\$ 5.3$

This class is easily seen to be independent of the choice of $x$. We also have the class

$$
\mu\left(\mathcal{C}_{g}^{1}, \hat{\xi}\right) \in H_{\text {êt }}^{1}\left(\mathcal{M}_{g}^{1}, \mathbb{L}_{\mathbb{Z}_{\ell}}\right)
$$

of the universal cycle $\mathcal{C}_{\xi}-\mathcal{C}_{\xi}^{-}$.

The $\ell$-adic Harris-Pulte Theorem is a direct consequence of the following result.

Theorem 8.1. The classes $\delta_{C, x}^{*} \hat{m}^{(\ell)}$ and $\mu\left(\mathcal{C}_{g}^{1}, \hat{\xi}\right)$ in $H_{\mathrm{êt}}^{1}\left(\mathcal{M}_{g}^{1}, \mathbb{L}_{\mathbb{Z}_{\ell}}\right)$ are equal.

Proof. First suppose that $K$ is a subfield of $\mathbb{C}$. Denote the algebraic closure of $K$ in $\mathbb{C}$ by $\bar{K}$. Proposition 6.4 comparison theorems and the commutativity of the diagram

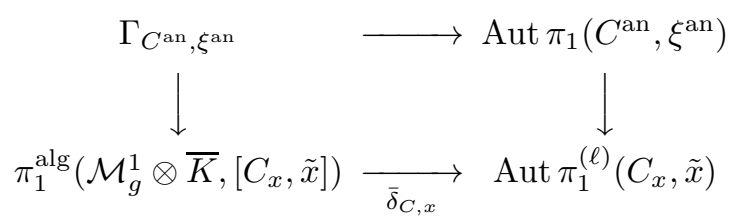

imply that

$$
\bar{\delta}_{C, x}^{*} \hat{m}^{(\ell)}=\mu\left(\mathcal{C}_{g}^{1}, \hat{\xi}\right) \in H_{\text {ét }}^{1}\left(\mathcal{M}_{g}^{1} \otimes \bar{K}, \mathbb{L}_{\mathbb{Z}_{\ell}}\right) .
$$

The result now follows from Corollary 6.7 The case of general $K$ follows from the case where $K \subset \mathbb{C}$ by standard techniques of GAGA.

The Harris-Pulte Theorem now follows by specialization:

Proof of Theorem 3 . As in the statement of the theorem, we suppose that $K$ is a subfield of $\mathbb{C}$ and that $(C, \xi)$ is a pointed curve over $K$. Choose a geometric point $x$ of Spec $K$. We shall identify $\pi_{1}^{(\ell)}\left(C_{x}, \tilde{x}\right)$ with $\pi_{1}^{(\ell)}\left(C^{\text {an }}, \xi^{\text {an }}\right)$. The class $m(C, \xi)$ is the pullback of the universal class $\hat{m}^{(\ell)}$ under the natural homomorphism

$$
\rho_{C, x}^{(\ell)}: G_{K} \rightarrow \operatorname{Aut} \pi_{1}^{(\ell)}\left(C^{\text {an }}, \xi^{\text {an }}\right) .
$$


Since the diagram

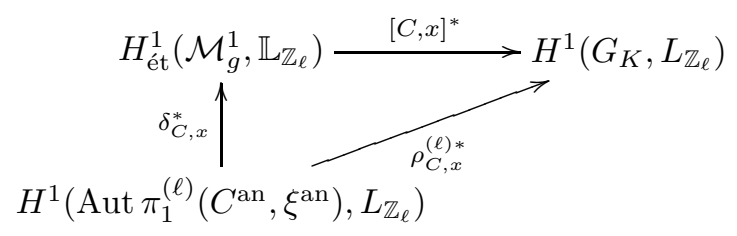

commutes, Proposition 4.1 implies that

$$
m(C, x)=\rho_{C, x}^{(\ell) *} \hat{m}^{(\ell)}=[C, x]^{*} \delta_{C, x}^{*} \hat{m}^{(\ell)}=[C, x]^{*} \mu\left(\mathcal{C}_{g}^{1}, \hat{\xi}\right)=\mu(C, x) .
$$

\section{The $\ell$-ADic Formulation}

As in previous sections, $C$ is a smooth projective curve of genus $\geq 3$ defined over the subfield $K$ of $\mathbb{C}, \bar{K}$ is its algebraic closure in $\mathbb{C}, \xi$ is a $K$-rational point of $C, x$ is the geometric point $\operatorname{Spec} \bar{K}$ of $\operatorname{Spec} K$ and $\tilde{x}$ is $\xi(x)$.

We have the truncations

$$
\rho_{C, \xi}^{(\ell), m}: G_{K} \rightarrow \operatorname{Aut}\left[\pi_{1}^{(\ell)}\left(C^{\mathrm{an}}, \xi^{\mathrm{an}}\right) / L^{m+1}\right]
$$

and

$$
\hat{\rho}_{C, \xi}^{(\ell), m}: \pi_{1}^{\mathrm{alg}}\left(\mathcal{M}_{g}^{1},\left[C_{x}, \tilde{x}\right]\right) \rightarrow \operatorname{Aut}\left[\pi_{1}^{(\ell)}\left(C^{\mathrm{an}}, \xi^{\mathrm{an}}\right) / L^{m+1}\right]
$$

of the monodromy representations $\rho_{C, \xi}^{(\ell)}$ and $\hat{\rho}_{C, \xi}^{(\ell)}$ defined in Section 3

In this section, we prove the following $\ell$-adic version of Theorem 1

Theorem 9.1. If the image of the $\ell$-adic cyclotomic character $\chi_{\ell}: G_{K} \rightarrow \mathbb{Z}_{\ell} \times$ is infinite, then the following four conditions are equivalent:

(a) $\operatorname{im} \rho_{C, \xi}^{(\ell), m}$ is an open subgroup of $\operatorname{im} \hat{\rho}_{C, \xi}^{(\ell), m}$ for all $m \geq 1$.

(b) $\operatorname{im} \rho_{C, \xi}^{(\ell), 2}$ is an open subgroup of $\operatorname{im} \hat{\rho}_{C, \xi}^{(\ell), 2}$.

(c) The image of $\rho_{C}^{(\ell), 1}: G_{K} \rightarrow \operatorname{GSp}\left(H_{\mathbb{Z}_{\ell}}\right)$ is open, and both the classes $\kappa(C, \xi)$ in $H^{1}\left(G_{K}, H_{\mathbb{Z}_{\ell}}\right)$ and $\nu(C)$ in $H^{1}\left(G_{K}, L_{\mathbb{Z}_{\ell}} / H_{\mathbb{Z}_{\ell}}\right)$ have infinite order.

(d) Any of the 3 equivalent conditions (i), (ii), (iii) of Theorem [1.

Clearly, (a) $\Longrightarrow$ (b) $\Longrightarrow$ (c) $\Longrightarrow$ (iii). To complete the proof, we now show that (iv) $\Longrightarrow$ (a), where statement (iv) is given in Section [7.1] This will follow directly from the following three results. We begin by showing that Zariski density of the action of $G_{K}$ on $H_{\mathbb{Q}_{\ell}}$ implies that the image of the Galois action on $H_{\mathbb{Z}_{\ell}}$ is open.

Lemma 9.2. If $\Gamma$ is a profinite group and $r: \Gamma \rightarrow \operatorname{GSp}\left(H_{\mathbb{Z}_{\ell}}\right)$ is a continuous homomorphism whose image in $\operatorname{GSp}\left(H_{\mathbb{Q}_{\ell}}\right)$ is Zariski dense, then the image of $r$ is open in $\operatorname{GSp}\left(H_{\mathbb{Z}_{\ell}}\right)$.

Proof. The image $G$ of $r$ is closed in $\operatorname{GSp}\left(H_{\mathbb{Z}_{\ell}}\right)$, and therefore an $\ell$-adic Lie group, 30. Its Lie algebra $\mathfrak{g}$ is thus an analytic Lie algebra over $\mathbb{Q}_{\ell}$. A result of Borel 3] 7.9 Cor.] implies that $[\mathfrak{g}, \mathfrak{g}]$ is the Lie algebra of an algebraic subgroup $H$ of $\operatorname{GSp}\left(H_{\mathbb{Q}_{\ell}}\right)$. This implies, via an argument using the exponential mapping, that the image of $[G, G]$ is open in $H\left(\mathbb{Q}_{\ell}\right)$. Since $G$ is Zariski dense in $\operatorname{GSp}\left(H_{\mathbb{Q}_{\ell}}\right)$, its commutator is Zariski dense in $\operatorname{Sp}\left(H_{\mathbb{Q}_{\ell}}\right)$, and hence $H$ must contain $\operatorname{Sp}\left(H_{\mathbb{Q}_{\ell}}\right)$. But $H$ is contained in the commutator of $\operatorname{GSp}\left(H_{\mathbb{Q}_{\ell}}\right)$, and so must be $\operatorname{Sp}\left(H_{\mathbb{Q}_{\ell}}\right)$. Since 
$G$ is dense in $\operatorname{GSp}\left(H_{\mathbb{Q}_{\ell}}\right)$, the image of the composite of $r$ with $\operatorname{GSp}\left(H_{\mathbb{Z}_{\ell}}\right) \rightarrow \mathbb{Z}_{\ell}{ }^{\times}$is infinite and therefore open. The result follows.

Lemma 9.3. Suppose that $\Gamma$ is a profinite group and that $U$ is a unipotent group over $\mathbb{Q}_{\ell}$. If $\Gamma \rightarrow U\left(\mathbb{Q}_{\ell}\right)$ is a Zariski dense homomorphism, then the image of $\Gamma$ is open in $U\left(\mathbb{Q}_{\ell}\right)$.

Proof. See [11, Lemma 7.5].

The weighted completion $\mathcal{G}\left(G_{K}\right)$ is an extension

$$
1 \rightarrow \mathcal{U}\left(G_{K}\right) \rightarrow \mathcal{G}\left(G_{K}\right) \rightarrow \mathrm{GSp} \rightarrow 1
$$

of proalgebraic groups, where $\mathcal{U}\left(G_{K}\right)$ is prounipotent.

Lemma 9.4. The image of $\operatorname{ker}\left\{G_{K} \rightarrow \mathrm{GSp}\left(\mathbb{Q}_{\ell}\right)\right\}$ in $\mathcal{U}\left(G_{K}\right)$ is Zariski dense.

Proof. By [11 Corollary 4.5], it is enough to show the vanishing of

$$
H^{1}\left(\operatorname{im}\left(G_{K}\right), V\right)
$$

for any negative irreducible GSp-module $V$ over $\mathbb{Q}_{\ell}$, where $\operatorname{im}\left(G_{K}\right)$ denotes the image of $G_{K}$ in $\mathrm{GSp}\left(\mathbb{Q}_{\ell}\right)$. By Lemma $9.2 \operatorname{im}\left(G_{K}\right)$ contains a non-torsion scalar matrix $a I$. Then, $a I$ is in the center of $\operatorname{im}\left(G_{K}\right)$, and hence acts trivially on $H^{1}\left(\operatorname{im}\left(G_{K}\right), V\right)$. On the other hand, $a I$ acts on $V$ by multiplication by a negative power of $a$. This shows that the cohomology is torsion, hence is trivial over $\mathbb{Q}_{\ell}$.

Proof of Theorem 9.1. Fix a positive integer $m$. Denote by $G$ the image of $\mathcal{G}\left(\mathcal{M}_{g}^{1}\right)$ in the algebraic group $\operatorname{Aut}\left[\mathfrak{p}(C, \xi) / L^{m+1}\right]$. The Zariski density of $\chi_{\ell}$ implies that $G$ is an extension

$$
1 \rightarrow U \rightarrow G \rightarrow \mathrm{GSp} \rightarrow 1,
$$

where $U$ is a unipotent group over $\mathbb{Q}_{\ell}$. Condition (iv) implies that $G$ is also the image of $\mathcal{G}\left(G_{K}\right)$ in $\operatorname{Aut}\left[\mathfrak{p}(C, \xi) / L^{m+1}\right]$. What we want to show is that the image of $G_{K}$ in $G\left(\mathbb{Q}_{\ell}\right)$ is open. Denote the kernel of $G_{K} \rightarrow \operatorname{GSp}\left(\mathbb{Q}_{\ell}\right)$ by $\Delta$. Since the image of $G_{K}$ in $\operatorname{GSp}\left(\mathbb{Q}_{\ell}\right)$ is open (Lemma 9.2), it suffices to show that $\Delta$ has open image in $U\left(\mathbb{Q}_{\ell}\right)$. By Lemma 9.3 it suffices to show that the image of $\Delta$ is Zariski dense in $U$. But this follows from Lemma 9.4 and the surjectivity of $\mathcal{U}\left(G_{K}\right) \rightarrow U$, which is a consequence of the surjectivity of $\mathcal{G}\left(G_{K}\right) \rightarrow G$.

\section{The Un-Pointed Case}

In this section, we briefly sketch the proof of Theorem 2 Since the arguments are very similar to those in the pointed case, the details are left to the reader. We also state its $\ell$-adic version and an un-pointed version of the Harris-Pulte Theorem that does not require the existence of a rational point. The proofs of these later two results are very similar to those in the pointed case and are left to the reader.

10.1. Monodromy representations. There are similar constructions to those in Section 3 in the non-pointed case, where Aut is replaced by Out. Specifically, suppose that $C \rightarrow B$ is a proper smooth family of genus $g$ curves defined over the field $K$ and that $x$ is a geometric point of $B$. Even though this may not have a section, there is still a natural homomorphism

$$
\rho_{C}: \pi_{1}^{\mathrm{alg}}(B, x) \rightarrow \text { Out } \pi_{1}^{\mathrm{alg}}\left(C_{x}\right),
$$


where $C_{x}$ denotes the geometric fiber of $C$ over $x$. This is constructed as the composite

$$
\pi_{1}^{\mathrm{alg}}(B, x) \stackrel{\simeq}{\longleftarrow} \pi_{1}^{\mathrm{alg}}(C, \tilde{x}) / \pi_{1}^{\mathrm{alg}}\left(C_{x}, \tilde{x}\right) \stackrel{\text { conjugation }}{\longrightarrow} \operatorname{Out} \pi_{1}^{\mathrm{alg}}\left(C_{x}, \tilde{x}\right)
$$

where $\tilde{x}$ is a geometric point of $C$ lying over $x$. This induces outer actions

$$
\rho_{C}^{*}: \pi_{1}^{\operatorname{alg}}(B, x) \rightarrow \text { Out } \pi_{1}^{*}\left(C_{x}\right)
$$

where $* \in\{\operatorname{alg},(\ell)$, un $\}$.

Denote the moduli stack of smooth projective curves of genus $g>1$ over $K$ by $\mathcal{M}_{g}$. It is defined over Spec $K$. Each pointed curve $C$ defined over an algebraically closed extension of $K$ determines a geometric point $[C]$ of $\mathcal{M}_{g}$; the fiber of the universal curve over this point may be identified with $C$. Associated to this geometric point is the monodromy representation

$$
\hat{\rho}_{C}: \pi_{1}^{\text {alg }}\left(\mathcal{M}_{g},[C]\right) \rightarrow \text { Out } \pi_{1}^{\text {alg }}(C) .
$$

This is called the universal monodromy representation for the curve $C$ for reasons that we now recall.

For each proper family $C \rightarrow B$ of smooth pointed curves of genus $g$ defined over $K$, there is a unique morphism $[C]: B \rightarrow \mathcal{M}_{g}$ that classifies the family. It takes the geometric point $x$ of $B$ to $\left[C_{x}\right]$. The family $C \rightarrow B$ is the pull-back of the universal curve $\mathcal{C}_{g} \rightarrow \mathcal{M}_{g}$ along $[C]$.

By universality, the fiber of $\mathcal{C}_{g} \rightarrow \mathcal{M}_{g}$ over $[C](x)$ can be identified naturally with $C_{x}$. Thus, the diagram

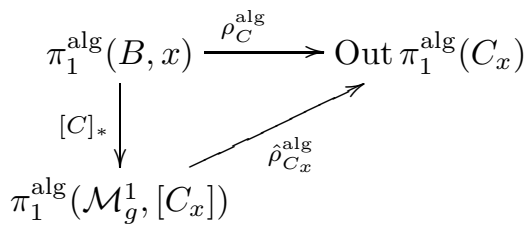

commutes. That is, the monodromy representation of $C \rightarrow B$ associated to the geometric point $x$ of $B$ factors through the universal monodromy representation (19) associated to $\left(C_{x}, \tilde{x}\right)$.

By functoriality, the universal monodromy representation (19) also has pro- $\ell$ and prounipotent incarnations:

$$
\hat{\rho}_{C}^{(\ell)}: \pi_{1}^{\text {alg }}\left(\mathcal{M}_{g},[C]\right) \rightarrow \operatorname{Out} \pi_{1}^{(\ell)}(C)
$$

and

$$
\hat{\rho}_{C}^{\mathrm{un}}: \pi_{1}^{\mathrm{alg}}\left(\mathcal{M}_{g},[C]\right) \rightarrow \operatorname{Out} \pi_{1}^{\mathrm{un}}(C)
$$

through which the representations (18) factor.

Now suppose that $K$ is a subfield of $\mathbb{C}$ and that $C$ is a curve defined over $K$. Let $\bar{K}$ be the algebraic closure of $K$ in $\mathbb{C}$. As in the pointed case, there is a natural isomorphism

$$
\widehat{\Gamma}_{C^{\mathrm{an}}} \cong \pi_{1}^{\mathrm{alg}}\left(\mathcal{M}_{g} \otimes \bar{K},[\bar{C}]\right),
$$

where $\bar{C}=C \otimes \bar{K}$, which is unique up to conjugation by an element of $\operatorname{Aut}\left(C^{\mathrm{an}}\right)$. Oda's result [28] implies that $\theta_{\bar{C}}$ is the completion of the tautological action

$$
\Gamma_{C^{\text {an }}} \rightarrow \text { Out } \pi_{1}\left(C^{\text {an }}\right) .
$$


10.2. The Johnson class. Let $\pi$ and $A$ be as in the beginning of Section 5.2 Since the inner automorphisms of $\pi$ act trivially on $H_{1}(\pi)$, the Johnson homomorphism induces homomorphisms

$$
\operatorname{Inn} \pi \rightarrow H_{1}(\pi) \rightarrow H_{1}(T) \rightarrow L_{A} .
$$

The image of the composition of these is well known and easily seen to be the standard copy of $H_{A}$ in $L_{A}$. It follows that if we write Out $\pi$ as an extension

$$
1 \rightarrow \mathrm{OT} \rightarrow \text { Out } \pi \rightarrow \operatorname{GSp}\left(H_{A}\right) \rightarrow 1,
$$

where $O T:=T / \operatorname{Inn} \pi$, then the Johnson homomorphism $\tau: T \rightarrow L_{A}$ induces a natural, $\operatorname{GSp}\left(H_{A}\right)$-invariant surjection

$$
H_{1}(O T) \rightarrow L_{A} / H_{A} .
$$

Arguing as in Section 5.3 one can prove the following analogue of Proposition 5.5 for outer actions.

Proposition 10.1. There is a unique class $\hat{n} \in H^{1}\left(\mathrm{Out} \pi, L_{A} / H_{A}\right)$ whose pullback to $H^{1}$ (Aut $\left.\pi, L_{A} / H_{A}\right)$ is the reduction of $\hat{m} \in H^{1}\left(\right.$ Aut $\left.\pi, L_{A}\right) \bmod H_{A}$.

In the $\ell$-adic case, we shall denote by $\hat{n}^{(\ell)}$ this universal characteristic class in $H^{1}$ (Out $\pi, L_{\mathbb{Z}_{\ell}} / H_{\mathbb{Z}_{\ell}}$ ).

For each curve $C$ over a field $K$ of characteristic 0, define

$$
n(C) \in H^{1}\left(G_{K}, L_{\mathbb{Z}_{\ell}} / H_{\mathbb{Z}_{\ell}}\right)
$$

to the the pullback of the universal class $\hat{n}^{(\ell)}$ along the natural action

$$
G_{K} \rightarrow \text { Out } \pi_{1}^{(\ell)}(C \otimes \bar{K}) \text {. }
$$

In the discrete case, we have Johnson's Theorem, which allows the computation of various cohomology groups of $\mathcal{M}_{g}^{\text {an }}$, and $\mathcal{M}_{g}$. The Torelli group $T_{S}$ of a compact oriented surface $S$ of genus $g$ is the kernel of the natural homomorphism $\Gamma_{S} \rightarrow$ Aut $H_{1}(S)$. Johnson's homomorphism for pointed surfaces induces an $\operatorname{Sp}\left(H_{\mathbb{Z}}\right)$ equivariant homomorphism

$$
\tau_{S}: H_{1}\left(T_{S}\right) \rightarrow L_{\mathbb{Z}} / H_{\mathbb{Z}} .
$$

A consequence of Johnson's Theorem (Thm. 6.1) is:

Corollary 10.2. If $g \geq 3$, then $\tau_{S}$ is surjective with finite kernel of exponent 2 .

10.3. Cohomology computations. Arguments similar to those in Section 6.3 can be used to prove the following result.

Proposition 10.3. If $g \geq 3$, then

(i) If $V$ is an irreducible rational representation of $\mathrm{Sp}\left(H_{\mathbb{Q}}\right)$ that is not isomorphic to $L_{\mathbb{Q}} / H_{\mathbb{Q}}$, then $H^{1}\left(\Gamma_{S}, V\right)$ vanishes.

(ii) The homomorphism

$$
H^{1}\left(\Gamma_{S}, L_{\mathbb{Z}} / H_{\mathbb{Z}}\right) \rightarrow H^{1}\left(\Gamma_{S, x}, L_{\mathbb{Z}} / H_{\mathbb{Z}}\right)
$$
induced by the canonical surjection $\Gamma_{S, x} \rightarrow \Gamma_{S}$ is an isomorphism.

(iii) The group $H^{1}\left(\Gamma_{S}, L_{\mathbb{Z}} / H_{\mathbb{Z}}\right)$ is freely generated over $\mathbb{Z}$ by a class whose image under the restriction mapping

$$
H^{1}\left(\Gamma_{S}, L_{\mathbb{Z}} / H_{\mathbb{Z}}\right) \rightarrow \operatorname{Hom}_{\mathrm{Sp}\left(H_{A}\right)}\left(H_{1}\left(T_{S}\right), L_{\mathbb{Z}} / H_{\mathbb{Z}}\right)
$$

is $2 \tau_{S}$. 
Note that the étale local system $\mathbb{L}_{\mathbb{Z}_{\ell}} / \mathbb{H}_{\mathbb{Z}_{\ell}}$ over $\mathcal{M}_{g}^{1}$ is actually the pullback of a local system over $\mathcal{M}_{g}$ for which we use the same notation. Specifically, this is the local system over $\mathcal{M}_{g}$ corresponding to the third fundamental representation of $\operatorname{GSp}\left(H_{\mathbb{Q}_{\ell}}\right)$.

Corollary 10.4. If $g \geq 3$, then the mapping

$$
H_{\text {ét }}^{1}\left(\mathcal{M}_{g}, \mathbb{L}_{\mathbb{Z}_{\ell}} / \mathbb{H}_{\mathbb{Z}_{\ell}}\right) \rightarrow H_{\text {ét }}^{1}\left(\mathcal{M}_{g}^{1}, \mathbb{L}_{\mathbb{Z}_{\ell}} / \mathbb{H}_{\mathbb{Z}_{\ell}}\right)
$$

induced by the projection $\mathcal{M}_{g}^{1} \rightarrow \mathcal{M}_{g}$ is an isomorphism. If $\mathbb{V}$ is the étale local system over $\mathcal{M}_{g}$ corresponding to an irreducible representation of $\operatorname{GSp}\left(H_{\mathbb{Q}_{\ell}}\right)$ that is not isomorphic to $\mathbb{L}_{\mathbb{Q}_{\ell}} / \mathbb{H}_{\mathbb{Q}_{\ell}}$ or some $\mathbb{Q}_{\ell}(n)$, then $H_{\text {ét }}^{1}\left(\mathcal{M}_{g}, \mathbb{V}\right)$ vanishes. In addition the structure morphism $\mathcal{M}_{g} \rightarrow \operatorname{Spec} K$ induces an isomorphism

$$
H^{1}\left(G_{K}, \mathbb{Q}_{\ell}(n)\right) \cong H_{\text {ét }}^{1}\left(\mathcal{M}_{g}, \mathbb{Q}_{\ell}(n)\right) \text {. }
$$

We shall denote the class in $H_{\text {ét }}^{1}\left(\mathcal{M}_{g}, \mathbb{L}_{\mathbb{Z}_{\ell}} / \mathbb{H}_{\mathbb{Z}_{\ell}}\right)$ that corresponds to $\nu\left(\mathcal{C}_{g}^{1}\right) \in$ $H_{\text {ét }}^{1}\left(\mathcal{M}_{g}^{1}, \mathbb{L}_{\mathbb{Z}_{\ell}} / \mathbb{H}_{\mathbb{Z}_{\ell}}\right)$ by $\nu\left(\mathcal{C}_{g}\right)$. Note that $H_{\text {êt }}^{1}\left(\mathcal{M}_{g}, \mathbb{L}_{\mathbb{Z}_{\ell}} / \mathbb{H}_{\mathbb{Z}_{\ell}}\right)$ is freely generated by it. This will allow us to extend, in the next section, the definition of $\nu(C)$ given in Section 10.4 to curves $C \rightarrow B$ without a $B$-rational point.

10.4. The invariant $\nu(C)$. The computations of the previous section can be used to define the invariant $\nu(C) \in H_{\text {êt }}^{1}\left(B, \mathbb{L}_{\mathbb{Z}_{\ell}} / \mathbb{H}_{\mathbb{Z}_{\ell}}\right)$ even when $C$ does not have any rational points, which proves Proposition 4.7

Suppose that $C \rightarrow B$ is a family of smooth projective curves of genus $\geq 3$ over $K$. It is classified by a morphism $[C]: B \rightarrow \mathcal{M}_{g}$. Define

$$
\nu(C) \in H_{\text {êt }}^{1}\left(B, \mathbb{L}_{\mathbb{Z}_{\ell}} / \mathbb{H}_{\mathbb{Z}_{\ell}}\right)
$$

to be the pullback $[C]^{*} \nu\left(\mathcal{C}_{g}\right)$ of the universal class. It follows from the results in the previous section that

$$
\nu(C)=\tilde{\nu}(C, \xi)
$$

when $C$ has a $B$-rational point $\xi$.

Theorem 2 can now be proved using arguments similar to those in Section 7 .

10.5. An un-pointed Harris-Pulte Theorem. There is a version of the $\ell$-adic Harris-Pulte Theorem for curves without any rational points.

Let $n(C) \in H^{1}\left(G_{K}, L_{\mathbb{Z}_{\ell}} / H_{\mathbb{Z}_{\ell}}\right)$ be the pullback of the universal class

$$
\hat{n}^{(\ell)} \in H^{1}\left(\operatorname{Out} \pi_{1}^{(\ell)}\left(C^{\text {an }}\right), L_{\mathbb{Z}_{\ell}} / H_{\mathbb{Z}_{\ell}}\right)
$$

along the homomorphism $G_{K} \rightarrow$ Out $\pi_{1}^{(\ell)}\left(C^{\text {an }}\right)$.

Theorem 10.5. If the genus of $C$ is $\geq 3$, then the classes $n(C)$ and $\nu(C)$ are equal in $H^{1}\left(G_{K}, L_{\mathbb{Z}_{\ell}} / H_{\mathbb{Z}_{\ell}}\right)$.

10.6. The $\ell$-adic formulation in the un-pointed case. As in previous sections, $C$ is a smooth projective curve of genus $\geq 3$ defined over the subfield $K$ of $\mathbb{C}, \bar{K}$ is its algebraic closure in $\mathbb{C}, x$ is the geometric point $\operatorname{Spec} \bar{K}$.

We have the truncations

$$
\rho_{C}^{(\ell), m}: G_{K} \rightarrow \operatorname{Out}\left[\pi_{1}^{(\ell)}\left(C^{\mathrm{an}}\right) / L^{m+1}\right]
$$

and

$$
\hat{\rho}_{C}^{(\ell), m}: \pi_{1}^{\mathrm{alg}}\left(\mathcal{M}_{g},[C \otimes \mathbb{C}]\right) \rightarrow \text { Out }\left[\pi_{1}^{(\ell)}\left(C^{\mathrm{an}}\right) / L^{m+1}\right]
$$

of the monodromy representations $\rho_{C}^{(\ell)}$ and $\theta_{C}^{(\ell)}$ defined in Section 10.1 
Theorem 10.6. If the image of the $\ell$-adic cyclotomic character $\chi_{\ell}: G_{K} \rightarrow \mathbb{Z}_{\ell}{ }^{\times}$is infinite, then the following four conditions are equivalent:

(a) $\operatorname{im} \rho_{C}^{(\ell), m}$ is an open subgroup of $\operatorname{im} \hat{\rho}_{C}^{(\ell), m}$ for all $m \geq 1$.

(b) $\operatorname{im} \rho_{C}^{(\ell), 2}$ is an open subgroup of $\operatorname{im} \hat{\rho}_{C}^{(\ell), 2}$.

(c) The image of $\rho_{C}^{(\ell), 1}: G_{K} \rightarrow \operatorname{GSp}\left(H_{\mathbb{Z}_{\ell}}\right)$ is open, and the class $\nu(C)$ in $H^{1}\left(G_{K}, L_{\mathbb{Z}_{\ell}} / H_{\mathbb{Z}_{\ell}}\right)$ has infinite order.

(d) Any of the 3 equivalent conditions (i), (ii), (iii) of Theorem 2 .

Acknowledgments. We would like to thank A. Tamagawa, S. Mochizuki, and K. Fujiwara for helpful conversations related to this work. We are also grateful to N. Kawazumi and S. Morita for correspondence related to the Magnus homomorphism.

\section{REFERENCES}

[1] S. Andreadakis: On the automorphisms of free groups and free nilpotent groups, Proc. London Math. Soc. 15 (1965), 239-268.

[2] S. Bloch, H. Esnault: The coniveau filtration and non-divisibility for algebraic cycles, Math. Ann. 304 (1996), 303-314.

[3] A. Borel: Linear algebraic groups, second edition, Graduate Texts in Mathematics 126, Springer-Verlag, New York, 1991.

[4] A. Borel: Stable real cohomology of arithmetic groups II, Manifolds and Groups, Papers in Honor of Yozo Matsushima, Progress in Mathematics 14, Birkhauser, Boston, 1981, 21-55.

[5] E. Colombo: The mixed Hodge structure on the fundamental group of hyperelliptic curves and higher cycles, J. Algebraic Geom. 11 (2002), 761-790

[6] P. Deligne, D. Mumford: The irreducibility of the space of curves of given genus, Inst. Hautes Études Sci. Publ. Math. No. 36 (1969), 75-109.

[7] B. Gross, C. Schoen: The modified diagonal cycle on the triple product of a pointed curve, Ann. Inst. Fourier 45 (1995), 649-679.

[8] A. Grothendieck: Revêtement Étales et Groupe Fondamental (SGA 1), Lecture Notes in Math. 224, Springer-Verlag 1971.

[9] R. Hain: Torelli groups and Geometry of Moduli Spaces of Curves, in Current Topics in Complex Algebraic Geometry (C. H. Clemens and J. Kollar, eds.) MSRI publications no. 28, Cambridge University Press, 1995, 97-143.

[10] R. Hain: Infinitesimal presentations of the Torelli groups, J. Amer. Math. Soc. 10 (1997), $597-651$.

[11] R. Hain, M. Matsumoto: Weighted completion of Galois groups and Galois actions on the fundamental group of $\mathbb{P}^{1}-\{0,1, \infty\}$, Compositio Math., to appear, math.AG/0006158

[12] R. Hain, M. Matsumoto: Tannakian fundamental groups associated to Galois groups, to appear in Galois groups and Fundamental Groups, MSRI, Leila Schneps (editor), math.AG/0010210

[13] R. Hain, M. Matsumoto: Completions of Arithmetic Mapping Class Groups, in preparation.

[14] R. Hain, D. Reed: On the Arakelov Geometry of Moduli Spaces of Curves, preprint 2002, math.AG/0211097

[15] U. Jannsen: Continuous Étale Cohomology, Math. Ann. 280 (1988), 207-245.

[16] U. Jannsen: Mixed motives and algebraic K-theory, Lecture Notes in Mathematics, 1400, Springer-Verlag, Berlin, 1990.

[17] D. Johnson: An abelian quotient of the mapping class group $\mathcal{I}_{g}$, Math. Ann. 249 (1980), 225-242.

[18] D. Johnson: The structure of the Torelli group. III. The abelianization of $\mathcal{T}$, Topology 24 (1985), 127-144.

[19] D. Johnson: A survey of the Torelli group, Low-dimensional topology (San Francisco, Calif., 1981), Contemp. Math., 20, Amer. Math. Soc., Providence, RI, 1983, 165-179.

[20] J. Labute: On the descending central series of groups with a single defining relation, J. Algebra 14 (1970) 16-23. 
[21] W. Magnus: Uber n-dimensional Gittertransformationen, Acta. Math. 64 (1934), 353-367.

[22] A. Malcev: Nilpotent torsion-free groups, (Russian) Izvestiya Akad. Nauk. SSSR. Ser. Mat. 13 (1949), 201-212.

[23] G. Margulis: Discrete subgroups of semisimple Lie groups, Ergebnisse der Mathematik und ihrer Grenzgebiete, 17, Springer-Verlag, Berlin, 1991.

[24] M. Matsumoto and A. Tamagawa: Mapping-class-group action versus Galois action on profinite fundamental groups, Amer. J. Math. 122 (2000), 1017-1026.

[25] J. Milne: Étale cohomology, Princeton Mathematical Series 33, Princeton University Press, 1980.

[26] Families of Jacobian manifolds and characteristic classes of surface bundles. II, Math. Proc. Cambridge Philos. Soc. 105 (1989), 79-101.

[27] S. Morita: The extension of Johnson's homomorphism from the Torelli group to the mapping class group, Invent. Math. 111 (1993), 197-224.

[28] T. Oda: Etale homotopy type of the moduli spaces of algebraic curves, in "Geometric Galois Actions 1", London Math. Soc. Lecture Note Series 242, 1997, 85-95.

[29] M. Pulte: The fundamental group of a Riemann surface: mixed Hodge structures and algebraic cycles, Duke Math. J. 57 (1988), 721-760.

[30] J.-P. Serre: Lie algebras and Lie groups, 1964 lectures given at Harvard University, Second edition, Lecture Notes in Mathematics, 1500, Springer-Verlag, Berlin, 1992.

[31] J. Tate: Relations between $K_{2}$ and Galois Cohomology, Invent. Math., 30 (1976), 257-274.

[32] V. Zoonekynd: The fundamental group of an algebraic stack, preprint, math.AG/0111071

Department of Mathematics, Duke University, Durham, NC 27708-0320

E-mail address: hain@math.duke.edu

Department of Mathematics, Hiroshima University, Hiroshima, 739-8526 JAPAN

E-mail address: m-mat@math.sci.hiroshima-u.ac.jp 